\title{
BMJ Open Risk factors, symptom reporting, healthcare-seeking behaviour and adherence to public health guidance: protocol for Virus Watch, a prospective community cohort study
}

Andrew Hayward, ${ }^{1}$ Ellen Fragaszy, ${ }^{2,3}$ Jana Kovar, ${ }^{1}$ Vincent Nguyen, ${ }^{1,2}$ Sarah Beale, ${ }^{1,2}$ Thomas Byrne, ${ }^{2}$ Anna Aryee, ${ }^{2}$ Pia Hardelid (1), ${ }^{4}$ Linda Wijlaars (1) , ${ }^{5,6}$ Wing Lam Erica Fong, ${ }^{2}$ Cyril Geismar, ${ }^{1,2}$ Parth Patel, ${ }^{2}$ Madhumita Shrotri, ${ }^{2}$ Annalan M D Navaratnam, ${ }^{1,2}$ Eleni Nastouli, ${ }^{5,7}$ Moira Spyer, ${ }^{5,7}$ Ben Killingley, ${ }^{8,9}$ Ingemar Cox, ${ }^{10}$ Vasileios Lampos, ${ }^{10}$ Rachel A McKendry, ${ }^{11}$ Yunzhe Liu, ${ }^{12}$ Tao Cheng, ${ }^{12}$ Anne M Johnson, ${ }^{13}$ Susan Michie, ${ }^{14}$ Jo Gibbs, ${ }^{13}$ Richard Gilson, ${ }^{13}$ Alison Rodger, ${ }^{13,15}$ Robert W Aldridge (10) ${ }^{2}$

To cite: Hayward A, Fragaszy E, Kovar J, et al. Risk factors, symptom reporting, healthcareseeking behaviour and adherence to public health guidance: protocol for Virus Watch, a prospective community cohort study. BMJ Open 2021;11:e048042. doi:10.1136/ bmjopen-2020-048042

- Prepublication history and supplemental material for this paper is available online. To view these files, please visit the journal online (http://dx.doi. org/10.1136/bmjopen-2020048042).

Received 22 December 2020 Revised 29 March 2021 Accepted 13 April 2021

Check for updates

(C) Author(s) (or their employer(s)) 2021. Re-use permitted under CC BY. Published by BMJ.

For numbered affiliations see end of article.

Correspondence to Dr Andrew Hayward; a.hayward@ucl.ac.uk

\section{ABSTRACT}

Introduction The coronavirus (COVID-19) pandemic has caused significant global mortality and impacted lives around the world. Virus Watch aims to provide evidence on which public health approaches are most likely to be effective in reducing transmission and impact of the virus, and will investigate community incidence, symptom profiles and transmission of COVID-19 in relation to population movement and behaviours.

Methods and analysis Virus Watch is a household community cohort study of acute respiratory infections in England and Wales and will run from June 2020 to August 2021. The study aims to recruit 50000 people, including 12500 from minority ethnic backgrounds, for an online survey cohort and monthly antibody testing using home fingerprick test kits. Nested within this larger study will be a subcohort of 10000 individuals, including 3000 people from minority ethnic backgrounds. This cohort of 10000 people will have full blood serology taken between October 2020 and January 2021 and repeat serology between May 2021 and August 2021. Participants will also post self-administered nasal swabs for PCR assays of SARS-CoV-2 and will follow one of three different PCR testing schedules based on symptoms. Ethics and dissemination This study has been approved by the Hampstead National Health Service (NHS) Health Research Authority Ethics Committee (ethics approval number 20/HRA/2320). We are monitoring participant queries and using these to refine methodology where necessary, and are providing summaries and policy briefings of our preliminary findings to inform public health action by working through our partnerships with our study advisory group, Public Health England, NHS and government scientific advisory panels.

\section{INTRODUCTION}

The COVID-19 pandemic has caused millions of deaths and impacted lives around the world with the closure of schools, workplaces

\section{Strengths and limitations of this study}

- Virus Watch is a large national household community cohort study of the occurrence of and risk factors for COVID-19 infection that aims to recruit 50000 people, including 12500 from minority ethnic backgrounds.

- Virus Watch is designed to estimate the incidence of PCR-confirmed COVID-19 in those with respiratory and non-respiratory presentations and the incidence of hospitalisation among PCR-confirmed COVID-19 cases.

- Virus Watch will measure effectiveness and impact of recommended COVID-19 control measures including testing, isolation, respiratory and hand hygiene measures, and social distancing on risk of respiratory infection.

- Only households with a lead householder able to speak English are able to take part in the study. Participant information sheets and consent forms are available in 9 languages but the study surveys are in English, limiting participation for non-English speaking households.

> Only households of up to six people were eligible for inclusion and they are also required to have access to an internet connection. These restrictions will limit the generalisability to large or multigenerational households, and those without access to the internet.

and limitations on freedom of movement. Vaccines and effective scalable treatments for COVID-19 have been developed and while these are rolled out across England and Wales we will need to rely on other measures to stop the spread of COVID-19. We will also require 
studies to examine their long-term effectiveness as they are implemented across England and Wales.

Governments, including those of the UK devolved nations, are adopting a wide range of control measures to limit the spread of infection. These include isolation of people with COVID-19 symptoms and their household contacts, widespread testing and contact tracing, digital contact tracing using mobile phone apps, broad social distancing measures and local control measures. Environmental cleaning, hand hygiene and face mask use are also advised.

Much of our current knowledge of COVID-19 comes from observations at the more severe end of the disease spectrum, in hospitalised patients and individuals who die having tested positive for the disease. ${ }^{1-3}$ Although largescale studies of prevalence of PCR positive infection and seroprevalence have been established, there is currently limited information on symptom profiles through the course of illness in non-hospitalised populations, children, social and behavioural risk factors for infection, strength and duration of immunity, household and community transmission risk, and population behaviours during periods of wellness and illness (including social contacts, use of public spaces, testing behaviours, isolation, mask use, hand and respiratory hygiene). This information can only be gathered accurately through prospective largescale community cohorts. Our experience of the Medical Research Council (MRC)/Wellcome Flu Watch study ${ }^{45}$ and the Economic and Social Research Council (ESRC) Bug Watch ${ }^{6}$ study has allowed us to rapidly establish a national household cohort study of 50000 individuals.

Virus Watch aims to provide evidence on which public health approaches are most likely to be effective in reducing the spread and impact of the virus and will investigate community incidence, symptom profiles and transmission of COVID-19 in relation to population movement and behaviour.

\section{METHODS AND ANALYSIS \\ Study design and setting}

Virus Watch is a household community cohort study of acute respiratory infections in England and Wales covering the second and potential subsequent waves of the COVID-19 pandemic. The study period will be from 1 June 2020 to 31 August 2021. The study aims to recruit 50000 individuals, including 12500 from minority ethnic backgrounds for an online survey cohort (study 1). Nested within this larger study will be a subcohort of 10000 individuals (study 2), including 3000 people from minority ethnic backgrounds. Participants in this laboratory subcohort will be selected based on their geographical distance from one of our bloodtaking clinics; either a $10 \mathrm{~km}$ radius from a clinic in cities or a $20 \mathrm{~km}$ radius in rural areas. Participants will be balanced to be representative of the UK population for sex, age and region. Figure 1 provides an overview of the study design.

Households self-select into the study if they live in England or Wales and all members of a household need to consent to take part in the study to meet our inclusion criteria (online supplemental appendix 1). Households need to have an internet connection on a phone, tablet or computer, email, and at least one adult household member that can read English.A household is defined as one or more people (not necessarily related) whose usual residence ( 4 days/week or more) is at the same address. These householders share cooking facilities, a living room or sitting room or dining area.

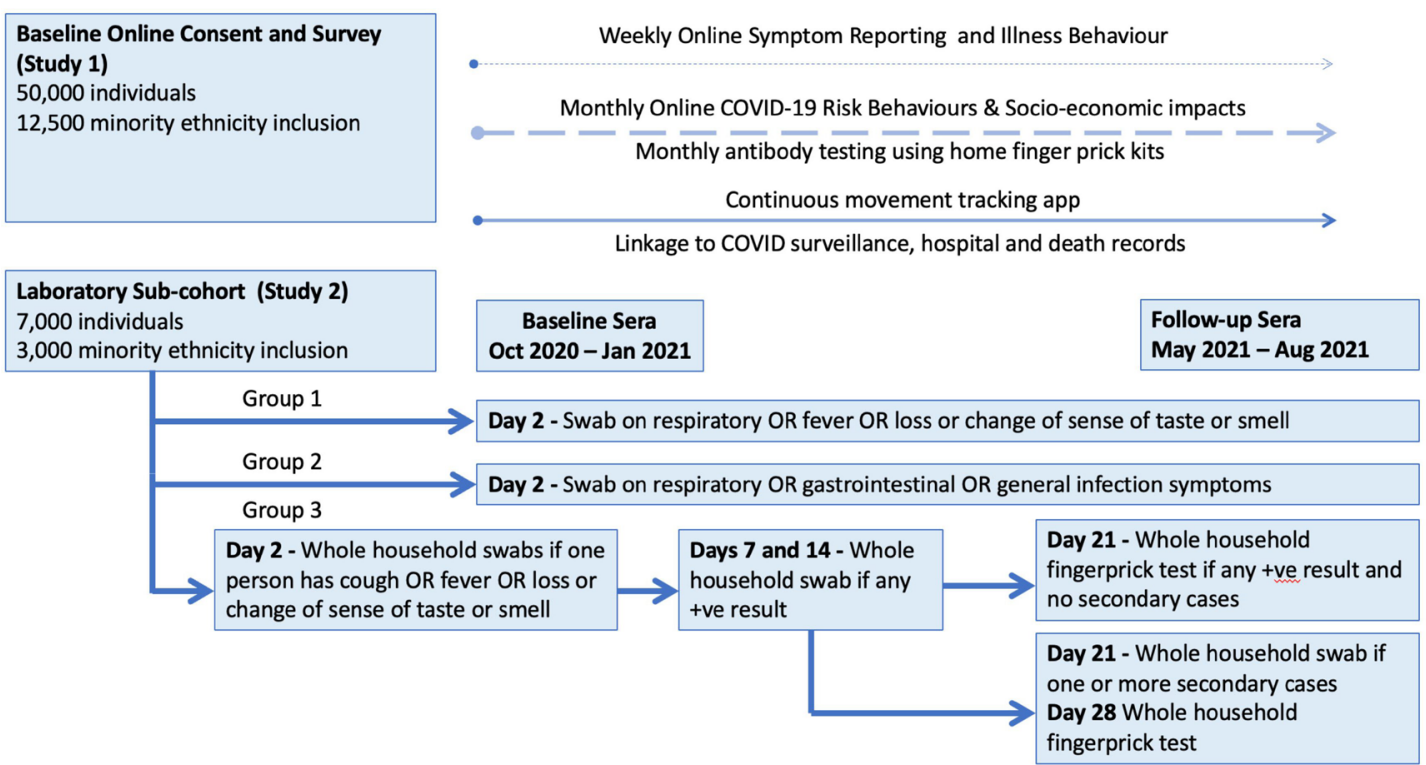

Figure 1 Overview of cohort recruitment, PCR swabbing schedules and data collection for the Virus Watch household community cohort study. 


\section{Primary outcomes}

Study 1: online survey cohort

1. Incidence of respiratory infection symptoms, including COVID-19 disease case definitions.

2. Effectiveness and impact of recommended COVID-19 control measures including testing, isolation, social distancing, respiratory and hand hygiene measures on risk of respiratory infection.

3. Frequency of adherence to public health recommendations for these control measures.

4. Proportion of community infections that result in hospital admissions and death.

5. Vaccine effectiveness against asymptomatic and symptomatic infections.

Study 2: laboratory testing subcohort

1. Incidence of PCR-confirmed COVID-19.

2. Incidence of PCR-confirmed COVID-19 in those with non-respiratory presentations.

3. Incidence of hospitalisation among PCR-confirmed COVID-19 cases.

4. Proportion of individuals with SARS-CoV-2 antibodies acquired through natural infection to pandemic coronavirus.

5. Proportion of individuals with cross-reacting antibodies to seasonal coronaviruses acquiring (or not) SARSCoV-2.

6. Household secondary attack rates.

7. Protective effect of antibodies on infection and reinfection as well as the severity and spectrum of presentation.

\section{Recruitment}

We will use the Royal Mail Post Office Address File to generate a list of residential address lists from which households can be sampled and sent Virus Watch recruitment postcards to. The proposed initial sample design is a single-stage stratified probability sample where implicit stratification is employed to benefit from the precision gains that stratified sampling can bring. Within each region, residential addresses are sorted by (A) quintiles of Index of Multiple Deprivation 2019, (B) within quintiles by local authorities, (C) postcodes, and (D) address. We will perform this in the nine government office regions of England as well as Wales (10 study regions in total).

We will assess recruitment rates and the representativeness of this initial sample following the mail out of 50000 postcards. If recruitment is lower than expected or under-representative of the national population, we will redesign our recruitment campaign to include a range of methods in order to build the cohort. This mixed recruitment strategy will be flexible and use a variety of methods including social media, study leaflet drops, text messaging, personalised letters and incentives. Social media adverts will be used to inform individuals about the study and direct them to our website, http:// ucl-virus-watch.net/, where they can read the participant information sheets and consent to taking part. Digital invitations will also be created for sharing via WhatsApp. Text messages and postal letters inviting patients from their general practitioner (GP) clinics will be organised via Local Clinical Research Networks. ${ }^{7}$ We will also work with trusted community partners and religious organisations to promote recruitment into the study.

In order for a household to be enrolled, they will require an internet connection (Wi-Fi, fixed or on a mobile phone), email address, and all household members must agree to take part. Households will nominate a lead householder who will submit study questionnaires. The lead householder will need to be able to read English to support other household members in survey completion. A household is defined as one or more people (not necessarily related) whose usual residence (4 days/week or more) is at the same address. These householders share cooking facilities, and may share a living room or sitting room or dining area if available. Households with more than six members will not be eligible for the studythis criterion was set due to limitations of the Research Electronic Data Capture (REDCap) survey infrastructure which did not function correctly when attempting to work with household sizes of greater than six during our pilot testing of the survey.

Virus Watch is powered for our primary aims in study 2 and the estimation of population-level symptomatic COVID-19 attack rate over time. Recruiting a cohort that is representative of the population is time consuming as it requires an initial invitation into a study followed by multiple follow-up contacts encouraging invited individuals to register. Given the urgency of the public health situation to roll out our study as quickly as possible, we chose a different approach whereby we recruit a large cohort of 50000 individuals and from within that cohort we select a subsample for the testing cohort (subcohort 1) which is representative of the population in terms of age, sex, ethnicity, region, household size and proportion of households with children. The larger cohort will be important in assessing rates and predictors of less frequent outcomes such as hospitalisation and death. Given recent information of marked ethnicity differences in mortality rates from COVID-19, we also chose to recruit an ethnicity sample designed to be sufficiently large to provide early indicators of whether these differential mortality rates are due to differences in disease incidence or in differences in severity or both.

\section{Power analysis}

The testing subcohort is powered for accurate weekly agespecific disease incidence rates to be measured assuming $20 \%-30 \%$ clinical attack rate over 18 weeks. With a clinical attack rate of $30 \%$ of whom $20 \%$ need hospitalisation and $0.5 \%$ die, we expect the following number of outcome events in our testing cohort of 10000 individuals in study 2: 3000 COVID-19 illnesses, 600 hospitalised cases and 15 deaths. At 1 month into the outbreak we would be able to detect a 1.7 -fold greater risk of disease in a population subgroup that constitutes one-fifth of the population, and 
by 2 months the detectable relative risk would be only 1.2. At 1 month we could detect a $4 \%$ hospital admission rate among cases with $95 \%$ CI of 0.5 to 6.8 , and by 2 months the CIs would narrow to 3.1 to 4.1 . We have used estimates of the expected number of events over time to provide an indication of the fact that the cohort is sufficiently large to provide valuable information through the course of the pandemic. Sample size calculations have been informed by a realistic assessment of what we can achieve based on our previous experience. ${ }^{46}$ For the serology cohort of 3000 people from minority ethnic backgrounds we assume a modest design effect due to household and geographical clustering, and 500 participants for six different minority ethnic backgrounds would enable the measurement of a cumulative incidence of $10 \%$ with $95 \%$ CIs of $3 \%$ by each minority ethnic group.

\section{Participant materials and incentives}

Participant information sheets will be held on our study website (these along with consent forms were translated into six languages and a further three languages were added from December 2020). In order to participate, the whole household must take part. Each adult participant will need to read through study information, and provide online informed consent for themselves and any children they are legally responsible for. Children aged 6-9 and 10-15 years, respectively, will also be asked to read through age-specific study participant information sheets and provide online informed assent. For children aged 5 and under, parents/guardians will consent on their behalf. Informed consent data will be securely stored in the University College London (UCL) Data Safe Haven which has been certified to the ISO27001 information security standard and conforms to the National Health Service (NHS) Digital's Data Security and Protection Toolkit. Local study teams will reconsent participants face to face, prior to undertaking blood sampling, and adult participants in study 2 will be offered a £10 voucher to reimburse travel costs, if required. From February 2021, invitation letters sent by GP clinics will include a $£ 20$ voucher for households who agree to take part in the study.

\section{Data collection and follow-up}

\section{Study 1: online survey cohort}

The online survey cohort will collect data and follow up participants through six different sources. Survey data will be collected using REDCap electronic data capture tools hosted on the UCL Data Safe Haven (online supplemental appendices 2-4). ${ }^{8}$ REDCap is a secure, web-based application for research studies. The UCL Data Safe Haven provides a technical solution for storing, handling and analysing identifiable data. It has been certified to the ISO27001 information security standard and conforms to NHS Digital's Data Security and Protection Toolkit .

1. Baseline survey. The lead householder will be asked to complete an online baseline survey for each member of their household. Information collected includes: demographics, occupation, income, ethnicity, country of birth, year of entry to UK, chronic medical conditions, medications, pregnancy status, vaccines, mode of transport to work, any previous contact with someone with COVID-19, previous symptoms of COVID-19like illness and infection prevention behaviours such as social distancing and hand hygiene.

2. Illness surveys. Participants will be followed up weekly via an email with a link to an illness survey. This is a weekly survey of the presence or absence of symptoms that could indicate COVID-19 disease including respiratory, general infection or gastrointestinal symptoms. During illness, prospective daily symptom recording, quality of life, health-seeking behaviour (NHS 111, GP in person, GP by phone, accident and emergency, pharmacy, hospital), treatments and NHS investigations will be recorded. This survey will also include any respiratory and hand hygiene measures, self-isolation, activities and social contact, travel and face mask use. Questions around behavioural interventions, such as mask wearing and social distancing, aim to reflect the context and frequency/degree to which behaviours are practised according to governmental and public health guidelines and relevant scientific literature. The survey includes questions to the household on activities undertaken in the week prior to symptom onset. The weekly survey will also be used to capture test results received from outside the study and requests to self-isolate, for example, via the UK Test-TraceIsolate system. The weekly survey will also ask about participants' COVID-19 vaccination uptake, including their date of vaccination, dose (ie, first or second) and which vaccine was administered.

3. Monthly surveys. A number of questions will be asked every month. The monthly surveys also provide flexibility to ask additional questions (eg, behavioural changes) to reflect any new government directives on social distancing, testing, contact tracing and vaccine delivery. Core questions will also allow us to follow up reasons for any non-response in a given month (eg, because of illness, hospitalisation or holiday). We will also ask about online health information seeking, social distancing, including recent (week before) contacts, activities, places visited and hand and respiratory hygiene. As with the weekly questionnaire, questions around behavioural practices will reflect governmental and public health guidelines and the scientific literature; monthly questionnaires will also investigate barriers and enablers to health-related behaviours using purpose-developed questionnaires based on the Capability, Opportunity, Motivation, Behaviour model. We will also ask about finances, employment and mental health to see how the COVID-19 response is affecting participants' well-being and ability to work. We will ask about access to healthcare for non-COVID-19 health problems to explore the indirect health impacts of the pandemic. We will ask about any COVID-19 PCR or antibody test results performed outside the study and not 
already reported through baseline surveys. We will ask about influenza vaccine uptake and COVID-19 vaccination intentions.

4. Data linkage. NHS Digital will undertake quarterly data linkage between cohort 1 and Hospital Episode Statistics (HES), which includes admitted patient and critical care episodes, outpatient department bookings and emergency care contacts. This linkage will also include Office for National Statistics mortality data, COVID-19 vaccination records and virology testing data routinely collected by Public Health England (PHE), Public Health Wales and the Department of Health and Social Care through 'Pillar 1' (testing in hospital patients and health and care workers) and 'Pillar 2' (community testing). These data sources will be linked to the cohort using name, NHS numbers, dates of birth and postal address. Identifying variables will be removed before the linked data are transferred back to UCL for analysis. These data linkages will continue for up to 5 years after the end of the study as we anticipate COVID-19 will become a recurring winter infection and we wish to understand its impact on health services in subsequent years. These linkage studies will identify any participants who have been admitted to hospital or died due to causes that could be directly or indirectly linked to the COVID-19 pandemic. Indirect causes include those related to limitations in healthcare access during the pandemic. Reductions in the use of routine health services will also be monitored via linkage to HES data.

5. Geolocation tracking. All adult participants will be asked about optional consent to use a secure geolocation tracking app (Tracker for ArcGIS) installed on their mobile phone for the duration of the study.

6. Monthly antibody testing using home fingerprick kits. Adults aged 18 years and over enrolled in the online survey cohort, with the exception of those in laboratory testing subcohort group 3, will be offered monthly antibody testing starting February 2021 and continuing until the end of the study, using home fingerprick kits for self-collection of capillary blood samples. Those aged under 18 and living with adults enrolled in monthly antibody testing will continue completing online surveys. Monthly antibody testing (FebruaryAugust 2021) will use Conformitè Europëenne (CE)marked at-home fingerprick kits designed to collect small-volume (400-600 $\mu \mathrm{L})$ capillary blood samples. Samples are self-collected by adult participants and returned to a United Kindom Accreditation Service (UKAS)-accredited laboratory via prepaid post, where they will be tested for anti-nucleocapsid and anti-spike antibodies using validated electrochemiluminescence immunoassays.

\section{Study 2: laboratory testing subcohort}

All participants agreeing to take part in the main cohort (study 1) will be asked to provide consent to be contacted and invited to participate in one of the three laboratory testing subgroups. This will enable a cohort of 10000 individuals selected from the main cohort of 50000 individuals to be maximally representative of the population of England and Wales. All participants taking part in study 2 will be asked to use the national test, trace and isolation system in addition to providing samples as part of Virus Watch.

Study 2 will consist of three groups that will follow different schedules of antibody testing and nasal/throat swabs for PCR testing.

\section{Group 1 ( $n=7000)$}

With data from this group we aim to identify infection in those with a wide range of respiratory symptoms. Participants will be asked to submit a nose/throat swab if they experience 2 consecutive days of: fever $\left(>37.8^{\circ} \mathrm{C}\right)$, feeling feverish, or new persistent cough, or loss or altered sense of smell or taste (COVID-19 suspected case definition), or shortness of breath, or ear pain or change in hearing, or sore throat, or sneezing, or blocked nose, or runny nose, or wheeze or sinus pain or congestion (other respiratory manifestations).

\section{Group 2 ( $n=1000)$}

This group aims to identify the importance of nonrespiratory presentations. Participants will be asked to submit a self-taken nasal/throat swab for PCR identification of COVID-19 and other respiratory viruses if:

- Either 2 consecutive days of respiratory symptoms (eg, cough, runny nose, sneezing, shortness of breath, sore throat, blocked nose, sinus pain or congestion, ear pain or change in hearing, wheezing, loss of or altered sense of taste or sense of smell).

- Or 2 consecutive days of gastrointestinal symptoms (eg, diarrhoea/loose stools, abdominal pain, nausea or vomiting, loss of appetite).

- Or 2 consecutive days of general infection symptoms (eg, feeling feverish, having a high temperature, feelings of severe unexplained tiredness, generalised muscle or joint aches).

\section{Group 3 ( $n=2000)$}

This group aims to identify the extent of household transmission. Participants will be asked to submit a nose/throat swab if they experience 2 consecutive days of cough or fever or loss of sense of taste or smell. Household contacts of the index case will also be asked to submit a swab on the same day whether or not they have symptoms.

If any of the swabs indicate SARS-CoV-2 infection, all household members will be asked to repeat the swab on day 7 and day 14. If there are no new SARS-CoV-2 cases in the household arising from swabs on days 7 and 14 (assumed secondary cases) then all household members will be asked to undertake a home fingerprick antibody test on day 21. If there is one or more secondary cases in the household then the entire household will be asked to take an additional swab on day 21 and then undertake the fingerprick antibody tests on day 28. 


\section{End of follow-up}

Online participant follow-up will end in August 2021 for households enrolled in monthly antibody testing, and in May 2021 for others, although depending on the progression of COVID-19, we may ask participants to continue in the study for longer. Participants will be sent an exit survey. Participants will be contacted to arrange a second blood sample collection from April 2021. Follow-up through data linkage with HES, COVID-19 vaccination records and mortality data will continue for 5 years after the end of the study.

\section{Laboratory testing}

Antibody testing

Study 2 will be using two different types of antibody tests. First, full blood serology will be taken between October 2020 and January 2021. We will use experienced healthcare professionals, including research nurses from the National Institute for Health Research Clinical Research Networks. ${ }^{7}$ Depending on local circumstances, visits to participants' homes to take blood may also be arranged. Children aged 15 years or less can opt out of having their blood taken but will be offered a fingerprick antibody test conducted by a healthcare worker instead. All participants from laboratory group 3 will additionally be offered a fingerprick antibody test at the same time as blood taking. From April 2021 until July 2021, we will invite all participants back for full blood tests or, for children who do not wish to have a full bleed, healthcare workerdelivered fingerprick-based antibody tests.

Families of children who have not been able to attend for a blood test, or for a healthcare worker-delivered fingerprick antibody test, will be provided with postal kits to perform these at home. We also plan to use fingerprick antibody testing where local clinics are no longer able to undertake full blood tests due to COVID-19 travel restrictions. Extremely clinically vulnerable participants will be sent home fingerprick tests instead of being asked to provide a serological sample.

\section{Virus detection}

Participants will post swab samples for PCR assays of SARS-CoV-2, and subsequent testing for influenza virus, seasonal coronavirus, rhinovirus and respiratory syncytial virus. When SARS-CoV-2 is identified we will also undertake whole-genome sequencing of the virus. Samples for COVID-19 diagnostics will be handled and processed according to the NHS and UCL guidance on sample handling during the COVID-19 pandemic.

COVID-19 PCR and serology results will be returned to participants via email message systems. These messages will include links to official support, information and advice from NHS and PHE as well as advice on how to interpret results based on current evidence. In laboratory group 3, where positive test results will trigger further testing of the household, the results email will also include details explaining the additional testing requests. We will be not asking for inconclusive PCR results to be repeated

\section{Statistical analysis}

Our primary analyses during the winter 2020/2021 season will focus on estimating age-specific weekly rates of symptoms and risk factors for PCR-confirmed COVID-19 illness and hospitalisation. For these analyses we will use Poisson regression models that account for clustering by household using robust SEs and we will explore the use of stratification or weighting of the sample by age and region as necessary to give nationally representative estimates. Weekly rates will be expressed per 100000 personweeks for ease of comparison with national surveillance data.

We will examine the proportion of the population infected during the first wave (eg, February-September 2020) and second and potential future pandemic waves. We will estimate the percentage of the population infected by calculating age and wave-specific rates of serological infection and PCR-confirmed disease per 100 person-seasons using Poisson regression with robust SEs to account for household-level clustering. A personseason will be defined by the epidemic curve in the cohort and therefore rates will account for differential follow-up time during each epidemic peak. In these analyses we will examine risk factors for infection, disease, disease severity and disease transmission.

We will estimate the proportion of serologically confirmed SARS-CoV-2 infections leading to symptomatic disease. First, we will calculate age-adjusted attributable rates of illness due to infection (subtracting rates of respiratory illness in non-seroconverters from those in seroconverters). Second, we will measure the proportion of seroconverters with PCR-confirmed COVID-19. Analyses plans will be developed prior to conducting all analyses.

We will estimate vaccine effectiveness against asymptomatic SARS-CoV-2 infections and against symptomatic COVID-19 using anti-nucleocapsid seroconversion, positive PCR testing and self-reported symptom data. We will use both time-to-event and test-negative analytical frameworks. Using quantitative antibody data, we will assess the dynamics of anti-spike antibodies over time and the relationship between antibody titres and the risk of infection.

While the study is being conducted, we will produce early, preliminary results and analyses for participants, the general public, government scientific advisory groups and policymakers in order to inform the public health response to the pandemic. These analyses will be reactive to the epidemiological circumstances and are therefore not defined in this protocol.

\section{Modelling}

We will build on our experience of working with PHE, Google and Microsoft to use anonymous national or subnational aggregate web search engine data $^{9}{ }^{10}$ to monitor the spreading of the disease. We will use our study data as ground truth to train real-time disease prevalence estimation algorithms. We will annotate Global Positioning System tracking data into standard categories including time at work and home, social venues, supermarkets, 
hospitals, GPs and transport mode for incorporation in classical epidemiological analyses. Integrating the linked survey data, we will develop a predictive spatiotemporal transmission model to investigate the impact of various social distancing strategies.

\section{Missing data}

We have several strategies that attempt to address the issue of missing data. First, we have sought to minimise the amount and impact of missing data for key outcomes and exposures through the study design. For example, for a number of our primary outcomes (PCR-confirmed illness, hospitalisation and death) and exposures (vaccination) we collect data both as self-reported and through data linkage with the relevant national data sets and registries. Second, we sought to minimise missing serological and Virus Watch specific swabbing outcomes in adults by making willingness to provide relevant specimens a prerequisite to study registration. Third, we know from our experience of previous community cohort studies of acute infections (Flu Watch ${ }^{4}$ and Bug Watch $^{6}$ ) that response to weekly surveys (where our symptom data are collected) is high at around $75 \%$, which we believe is achieved by keeping these weekly data collections simple and quick to complete. We have aimed to replicate this approach in Virus Watch. Fourth, for important missing baseline demographic data (eg, age and sex) we have created follow-up surveys to try and collect missing data at a later point in time. Fifth, where necessary, we will address missing data in our analyses and use multiple imputation methods if appropriate.

\section{Patient and public involvement}

Due to the urgent nature of this study, we did not involve participants in its original design. We have previously conducted patient and public involvement to support similar community cohort studies of acute infections using similar methodologies. We have engaged the Young Persons Advisory Group for research at Great Ormond Street Hospital to provide feedback on our Children's Participant Information Sheets. We have worked with the Race Equality Foundation and Doctors of the World in advising on the inclusion of people from minority ethnic backgrounds in Virus Watch and have set up an advisory group to inform the ongoing design and dissemination of health equity aspects of Virus Watch. They were not asked to assess the burden of the intervention and time required to participate in the research due to the urgent nature of setting the study up. This advisory group (consisting of lay members of the public, community leaders, charities and policy organisations who will be reimbursed for their time) will guide our health equity analyses and steer us on their implications for people, communities and policy. The advisory group will also help us prioritise what information and results to share, when and in what format.

\section{ETHICS AND DISSEMINATION}

This is a national study that has been approved by the Hampstead NHS Health Research Authority Ethics Committee (ethics approval number 20/HRA/2320). The study is compliant with the requirements of General Data Protection Regulation (2016/679) and the Data Protection Act (2018). All investigators and study site staff will comply with the requirements of the General Data Protection Regulation (2016/679) with regard to the collection, storage, processing and disclosure of personal information, and will uphold the Act's core principles.

We will provide opportunities for survey participants to comment on survey methodology in the first monthly survey and consider revisions based on this. We are also monitoring participant queries through our study email address and using these to refine methodology where necessary.

\section{Data sharing and access}

We aim to share aggregate data from this project on our website and via a 'Findings so far' section on our website-https://ucl-virus-watch.net/. We will also be sharing individual record-level data with personal identifiers removed on a research data-sharing service such as the Office for National Statistics Secure Research Service. ${ }^{11}$ In sharing the data we will work within the principles set out in the UK Research and Innovation (UKRI) guidance on best practice in the management of research data. ${ }^{12}$ Access to use of the data while research is being conducted will be managed by the chief investigators (AH and RWA) in accordance with the principles set out in the UKRI guidance on best practice in the management of research data. It is the intention that the data arising from this research will initially be collected, cleaned and validated by the UCL research team and once this has been completed will be shared for wider use. We aim to make subsets of the data more rapidly available both on our study website and via the public-facing dashboard during the ongoing phase of data collection. In line with Principle 5 of the UKRI guidance on best practice in the management of research data, we plan to release data in batches as they become available or as updated results are published. Individual record data linked using NHS Digital will not be shared, only aggregated results. HES and mortality data may be obtained from a third party and are not publicly available. These data are owned by a third party and can be accessed by researchers applying to the Health and Social Care Information Centre for England. We will put analysis code on publicly available repositories to enable their reuse.

\section{Author affiliations}

${ }^{1}$ Institute of Epidemiology and Health Care, University College London, London, UK ${ }^{2}$ Centre for Public Health Data Science, Institute of Health Informatics, University College London, London, UK

${ }^{3}$ Department of Infectious Disease Epidemiology, LSHTM, London, UK ${ }^{4}$ Centre for Paediatric Epidemiology and Biostatistics, Institute of Child Health, University College London, London, UK

${ }^{5}$ Population, Policy and Practice, University College London, London, UK 
${ }^{6}$ Primary Care and Population Health, University College London, London, UK

${ }^{7}$ Francis Crick Institute, London, UK

${ }^{8}$ University of Nottingham School of Medicine, Nottingham, UK

${ }^{9}$ University College London Hospital, London, UK

${ }^{10}$ Department of Computer Science, University College London, London, UK

${ }^{11}$ London Centre for Nanotechnology and Division of Medicine, University College

London, London, UK

${ }^{12}$ SpaceTimeLab, Department of Civil, Environmental and Geomatic Engineering,

University College London, London, UK

${ }^{13}$ Institute for Global Health, University College London, London, UK

${ }^{14}$ Centre for Behaviour Change, University College London, London, UK

${ }^{15}$ Royal Free London NHS Foundation Trust, London, UK

Twitter Linda Wijlaars @epi_counts, Eleni Nastouli @Eleni Nastouli and Robert W Aldridge @rob_aldridge

Contributors Conceptualisation: AH, EF, JK, PH, EN, BK, IC, VL, RAM, TC, AMJ, SM, JG, RG, AR, RWA. Investigation, methodology: all authors. Project administration: $A H$ EF, JK, VN, SB, TB, AA, PH, LW, WLEF, CG, PP, MSh, AMDN, EN, MSp, RWA. Writingoriginal draft preparation: all authors. Software: VN, TB, SB, RWA. Resources: AH, EF, JK, PH, EN, BK, IC, VL, RAM, TC, YL, AMJ, SM, JG, RG, AR, RWA. Writing-review and editing: all authors.

Funding The research costs for the study have been supported by the MRC Grant Ref: MC PC 19070 awarded to UCL on 30 March 2020 and MRC Grant Ref: MR/ V028375/1 awarded on 17 August 2020. The study also received $\$ 15000$ of Facebook advertising credit to support a pilot social media recruitment campaign on 18 August 2020.

Competing interests AH serves on the UK New and Emerging Respiratory Virus Threats Advisory Group. AMJ was a governor of Wellcome Trust from 2011 to 2018 and is chair of the Committee for Strategic Coordination for Health of the Public Research.

Patient consent for publication Not required.

Provenance and peer review Not commissioned; externally peer reviewed.

Supplemental material This content has been supplied by the author(s). It has not been vetted by BMJ Publishing Group Limited (BMJ) and may not have been peer-reviewed. Any opinions or recommendations discussed are solely those of the author(s) and are not endorsed by BMJ. BMJ disclaims all liability and responsibility arising from any reliance placed on the content. Where the content includes any translated material, BMJ does not warrant the accuracy and reliability of the translations (including but not limited to local regulations, clinical guidelines, terminology, drug names and drug dosages), and is not responsible for any error and/or omissions arising from translation and adaptation or otherwise.

Open access This is an open access article distributed in accordance with the Creative Commons Attribution 4.0 Unported (CC BY 4.0) license, which permits others to copy, redistribute, remix, transform and build upon this work for any purpose, provided the original work is properly cited, a link to the licence is given, and indication of whether changes were made. See: https://creativecommons.org/ licenses/by/4.0/

\section{ORCID iDs}

Pia Hardelid http://orcid.org/0000-0002-0154-1306

Linda Wijlaars http://orcid.org/0000-0003-1222-2922

Robert W Aldridge http://orcid.org/0000-0003-0542-0816

\section{REFERENCES}

1 Official UK coronavirus dashboard. Available: https://coronavirus. data.gov.uk/ [Accessed 30 Nov 2020].

2 Statistics. COVID-19 daily deaths. Available: https://www.england. nhs.uk/statistics/statistical-work-areas/covid-19-daily-deaths/ [Accessed 30 Nov 2020].

3 Coronavirus (COVID-19). Available: https://www.ons.gov.uk/peop lepopulationandcommunity/healthandsocialcare/conditionsanddis eases [Accessed 30 Nov 2020].

4 Fragaszy EB, Warren-Gash C, Wang L, et al. Cohort profile: the flu Watch study. Int J Epidemiol 2017;46:e18.

5 Hayward AC, Fragaszy EB, Bermingham A, et al. Comparative community burden and severity of seasonal and pandemic influenza: results of the flu Watch cohort study. Lancet Respir Med 2014;2:445-54

6 Smith CM, Conolly A, Fuller C, et al. Symptom reporting, healthcareseeking behaviour and antibiotic use for common infections: protocol for bug Watch, a prospective community cohort study. BMJ Open 2019;9:e028676.

7 Clinical Research Network. Available: https://www.nihr.ac.uk/explorenihr/support/clinical-research-network.htm [Accessed 30 Nov 2020].

8 Harris PA, Taylor R, Thielke R, et al. Research electronic data capture (REDCap)-a metadata-driven methodology and workflow process for providing translational research informatics support. J Biomed Inform 2009;42:377-81.

9 Wagner M, Lampos V, Cox IJ, et al. The added value of online usergenerated content in traditional methods for influenza surveillance. Sci Rep 2018;8:13963.

10 Lampos V, Majumder MS, Yom-Tov E. Tracking COVID-19 using online search, 2020. Available: http://arxiv.org/abs/2003.08086v10

11 Accessing secure research data as an accredited researcher. Available: https://www.ons.gov.uk/aboutus/whatwedo/statistics/ requestingstatistics/approvedresearcherscheme [Accessed 12 Mar 2021].

12 UKRI. Guidance on best practice in the management of research data. Available: https://www.ukri.org/wp-content/uploads/2020/10/ UKRI-020920-GuidanceBestPracticeManagementResearchData.pdf 


\section{Appendix 1 - Study inclusion and exclusion criteria:}

Inclusion:

- Households self-select into the study.

- Participants need to join as a household (all must take part).

- They need to have internet connection on a phone, tablet or computer, email and at least one adult that can read English.

Exclusion Criteria

We will exclude participants if:

- Number of householders exceeds 6 .

- Those without internet connection on a phone, tablet or computer, or an email address available to them as they will be unable to register

- There is no adult in the household who can read English (from March 2021 this will no longer be an exclusion criteria)

- A household is defined as one or more people (not necessarily related) whose usual residence (4days/week or more) is at the same address. These householders share

- cooking facilities, a living room or sitting room or dining area. 


\title{
Confidential
}

\section{Baseline for Lead Householder}

\author{
Baseline Survey for [hh1_fname] [hh1_sname] \\ alive
}

The baseline survey collects some basic information about each person participating in Virus Watch. We will ask about each household member in turn, please ask each adult to complete their own information and help children to complete their sections or complete them for them
[hh1_fname] [hh1_sname]: At birth you were described as?
Male
Female
Intersex
$\bigcirc$ Prefer not to say

[hh1_fname] [hh1_sname]: Do you know your NHS Number?

This can usually be found on an NHS letter
OYes
No

[hh1_fname] [hh1_sname]: What is your NHS Number?

[hh1_fname] [hh1_sname]: Where is your place of Birth?

United Kingdom
Other

[hh1_fname] [hh1_sname]: When did you first come to live in the UK (approximately)?

DD/MM/YYYY 


\section{Confidential}

[hh1_fname] [hh1_sname]: Please specify where you were born:
Afghanistan
Albania

Algeria

American Samoa

Andorra

Angola

Anguilla

Antarctica

Antigua And Barbuda

Argentina

Armenia

Aruba

Australia

Austria

Azerbaijan

Bahamas

Bahrain

Bangladesh

Barbados

Belarus

Belgium

Belize

$\bigcirc$ Benin

Bermuda

Bhutan

Bolivia

Bosnia And Herzegovina

Botswana

Bouvet Island

Brazil

$\bigcirc$ British Indian Ocean Territory

Brunei Darussalam

Bulgaria

Burkina Faso

Burundi

Cambodia

Cameroon

Canada

Cape Verde

$\bigcirc$ Cayman Islands

Central African Republic

Chad

$\bigcirc$ Chile

$\bigcirc$ China

Christmas Island

Cocos (keeling) Islands

Colombia

Comoros

Congo

Congo, The Democratic Republic Of The

Cook Islands

Costa Rica

Cote D'ivoire

Croatia

Cuba

Cyprus

Czech Republic

Denmark

Djibouti

Dominica

Dominican Republic

East Timor

Ecuador

Egypt

El Salvador

Equatorial Guinea

Eritrea

Estonia

Ethiopia

projectredcap.org 


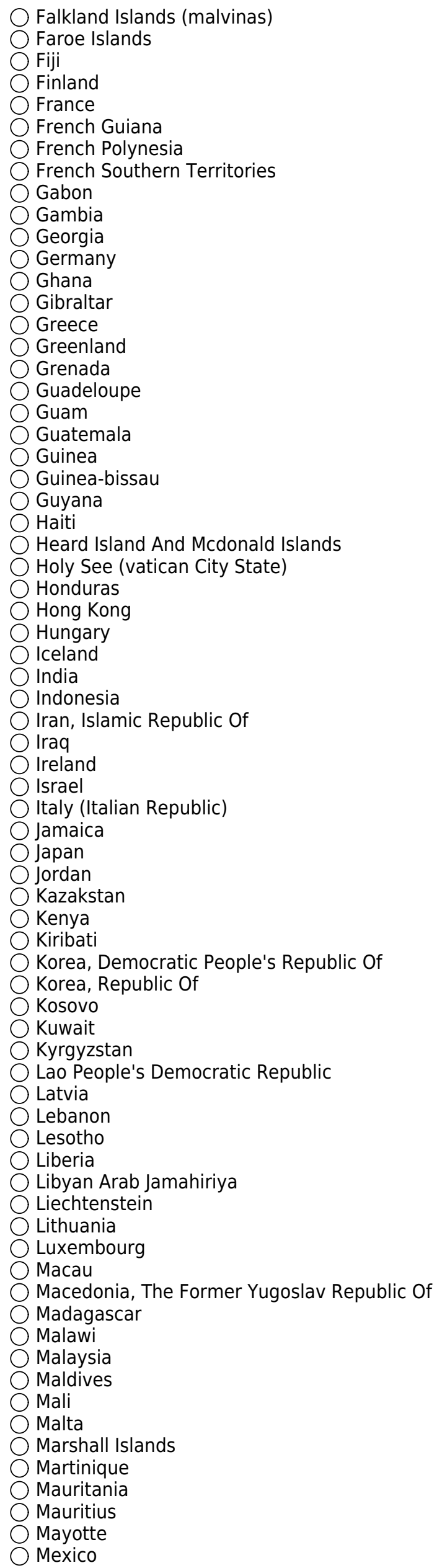




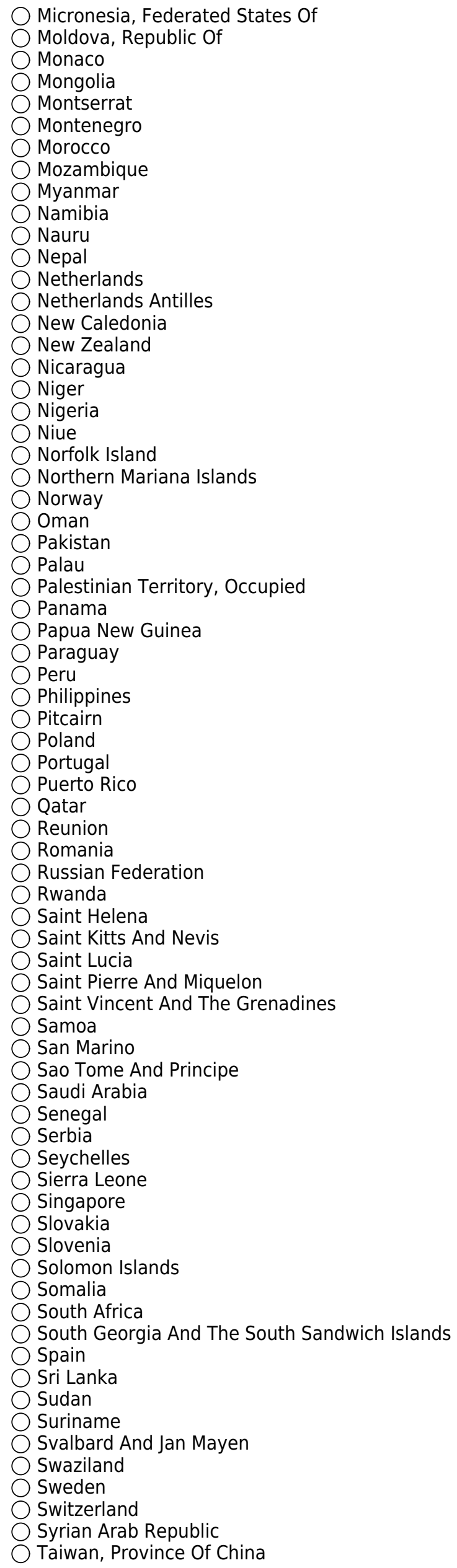




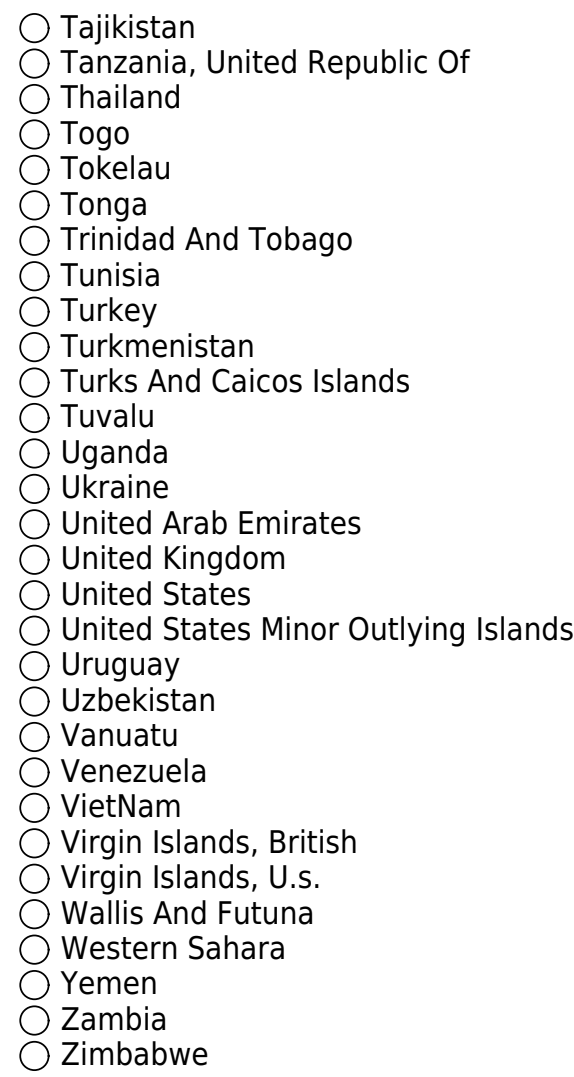

[hh1_fname] [hh1_sname]: What is your ethnic group?

White - English/ Welsh/ Scottish/ Northern Irish/ British

White - Irish

White - Gypsy or Irish Traveller

Any other white background (please describe)

Asian/ Asian British - Indian

Asian/ Asian British - Pakistani

Asian/ Asian British - Bangladeshi

Asian/ Asian British - Chinese

$\bigcirc$ Any other Asian/ Asian British background (please describe)

Black African

Black Caribbean

Any other Black/ African/ Caribbean background (please describe)

Arab

Any other ethnic group (please describe)

Mixed/ multiple ethnic groups - White and Black Caribbean

$\bigcirc$ Mixed/ multiple ethnic groups - White and Black African

Mixed/ multiple ethnic groups - White and Asian

Any other mixed/ multiple ethnic background (please describe)

$\bigcirc$ Prefer not to say

[hh1_fname] [hh1_sname]: Please describe your ethnic group:

[hh1_fname] [hh1_sname]: Are you pregnant?

Yes

No 


\title{
Confidential
}

What trimester of pregnancy are you in?

\author{
Less than 12 Weeks \\ 12 Weeks to 24 Weeks \\ More than 24 weeks
}

\section{Contact details}

[hh1_fname] [hh1_sname]: What is your mobile phone number?

This is so we can call you to make blood taking appointments and send your test results if you are selected for the swabbing part of the study.

If you do not have a mobile phone, please enter your landline phone number, and we will seek alternative arrangements to send your results if you are selected for the swabbing part of the study.

\section{Address Line 1:}

This is to send you swabs (if you are chosen by the study team to partake in the swabbing study)

\section{Address Line 2:}

Address Line 3:

Post Code: 


\section{Confidential}

\section{Medical Background for [hh1_fname] [hh1_sname]}

People's health can influence the severity of COVID illness, we want to find out more about this.

[hh1_fname] [hh1_sname]: Please provide your general practitioner's details:

This will allow us to link to your information to hospital records more accurately.

Name of Surgery:

Address Line 1:

Address Line 2:

Address Line 3:

Post Code:

[hh1 fname] [hh1 sname]: Has a doctor or other health professional ever told you that you have any of the following conditions?

Please select all that apply.
Asthma

Arthritis

Congestive heart failure

Coronary heart disease

Angina

Heart attack or myocardial infarction

Stroke

Emphysema

Chronic bronchitis

COPD (Chronic Obstructive Pulmonary Disease)

Cystic fibrosis

Hypothyroidism or an under-active thyroid

Any kind of liver condition

Cancer or malignancy

Insulin treated diabetes

Other diabetes

Epilepsy

$\square$ High blood pressure/hypertension

$\square$ An emotional, nervous or psychiatric problem

$\square$ Multiple Sclerosis

HIV

Chronic kidney disease

$\square$ Conditions affecting the brain and nerves, such as

Parkinson's disease, motor neurone disease,

multiple sclerosis (MS), a learning disability or cerebral palsy

$\square$ Problems with your spleen or you've had your spleen removed

$\square$ Sickle cell disease

$\square$ Other long standing/chronic condition

$\square$ None of these

[hh1_fname] [hh1_sname]: Please specify: 


\section{Confidential}

[hh1_fname] [hh1_sname]: What type of cancer or malignancy was that?

Please select all that apply

$\square$ Bowel/colorectal
$\square$ Lung
$\square$ Breast
$\square$ Prostate
$\square$ Liver
$\square$ Skin cancer or melanoma
$\square$ Blood or bone marrow cancer, such as leukaemia
$\square$ Other

[hh1_fname] [hh1_sname]: What type of cancer or malignancy was that? Please select all that apply [hh1_fname] [hh1_sname]: Has a doctor or other health professional ever told you that you have any of these conditions?

Please select all that apply

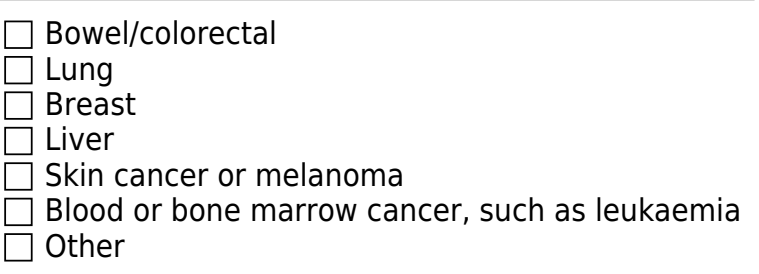

$\square$ Asthma

$\square$ Cystic fibrosis

$\square$ Insulin treated diabetes

$\square$ Epilepsy

$\square$ Conditions affecting the brain and nerves, such as Parkinson's disease, motor neurone disease, multiple sclerosis (MS), a learning disability or cerebral palsy

$\square$ Sickle cell disease

Other long standing/chronic condition

None of these

$\begin{array}{ll}\text { [hh1_fname] [hh1_sname] : Have you received a letter } & \bigcirc \text { Yes } \\ \text { from the NHS, saying that "the NHS has identified you } & \bigcirc \text { No }\end{array}$

as someone at risk of severe illness if you catch

coronavirus, because you have an underlying disease or

health condition that means if you catch the virus,

you are more likely to be admitted to hospital than

others"?

[hh1_fname] [hh1_sname] : Do you know your height and weight?

Yes

No
[hh1_fname] [hh1_sname]: Do you know your height in imperial (feet and inches) or metric (centimetres)?
Please select the unit you prefer if you know both

[hh1_fname] [hh1_sname]: What is your height in centimetres $(\mathrm{cm})$ ?

Please enter digits only, e.g. '5' and not 'five'

[hh1_fname] [hh1_sname]: How many feet tall are you (rounded down)?

Please enter the feet component of your height. For example if you're 5 foot 4 , please enter 5

[hh1_fname] [hh1_sname]: How many inches tall are you above your feet value?

Please enter the inches component of your height. For example if you're 5 foot 4 , please enter 4 


\title{
Confidential
}

\begin{abstract}
[hh1_fname] [hh1_sname]: Do you know your weight in imperial (stone and pounds-lbs) or metric(kilograms)?

Imperial (stone and pounds)

Please select the unit you prefer if you know both

[hh1_fname] [hh1_sname]: How much do you weigh in kilograms $(\mathrm{kg})$ ?

Please enter digits only, e.g. '5' and not 'five'

[hh1_fname] [hh1_sname]: What is your weight in stone, rounded down?

For example if you are 8 stone, 10 pounds, please enter 8 . If you do not use stone, please feel free to leave this blank and enter your weight fully in pounds

[hh1_fname] [hh1_sname]: How much do you weight in pounds (lbs) (above your stone weight)?

For example, if you are 8 stone, 10 pounds, please

enter 10.If you did not enter a value for stone,

please enter your weight fully in lbs here

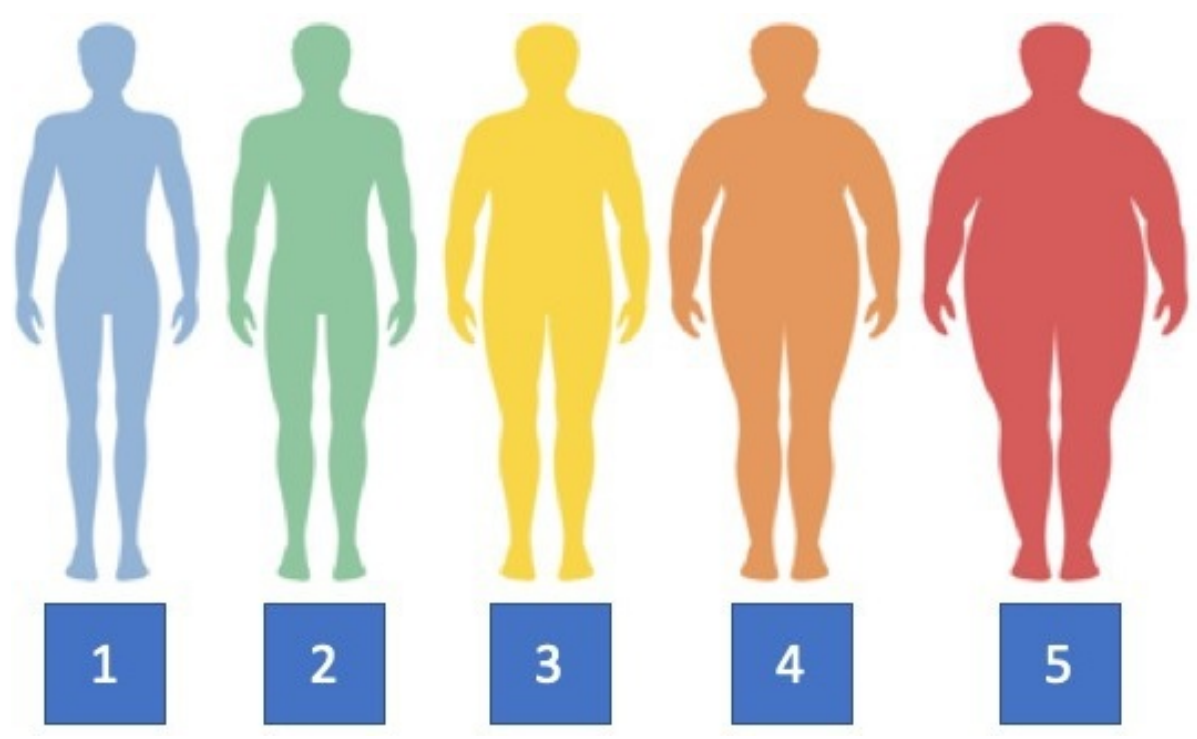




\section{Confidential}
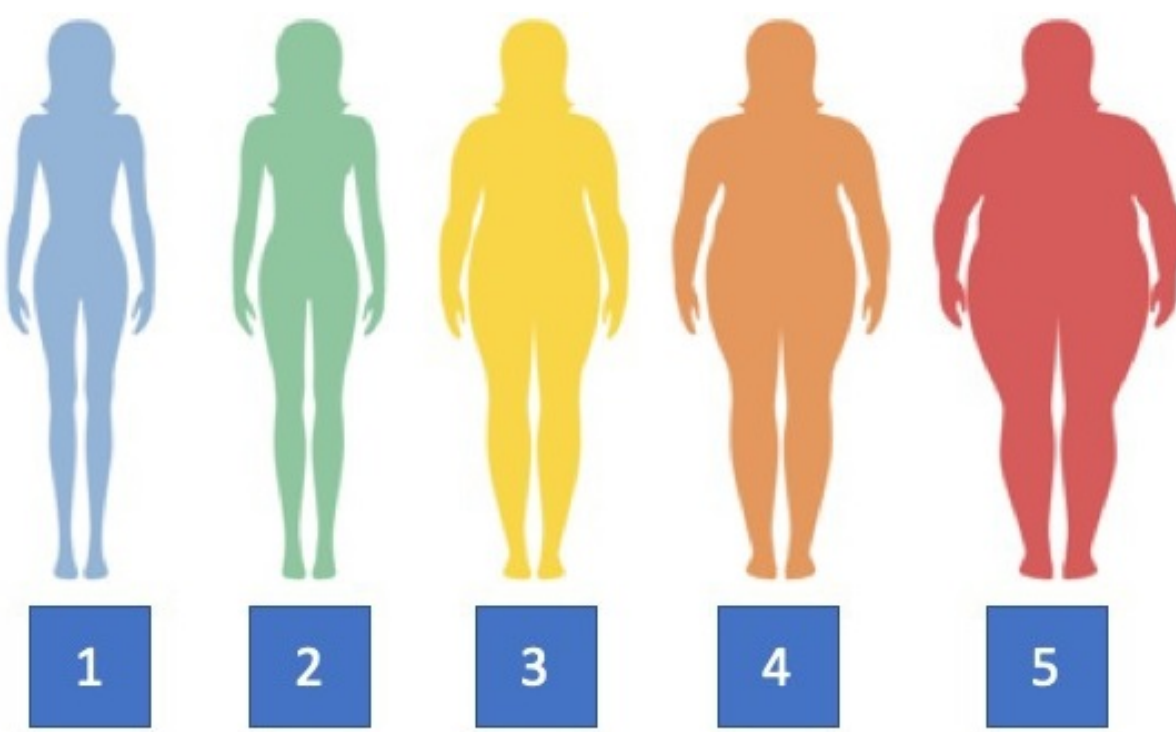

[hh1_fname] [hh1_sname]: Referring to the illustration above, which body shape bests describes your body

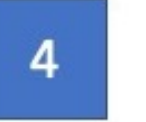
shape?
$\bigcirc 1$
$\bigcirc 2$
3
$\bigcirc 4$
5

HairSex hormones that affect male pattern baldness may also affect COVID severity

\section{1}

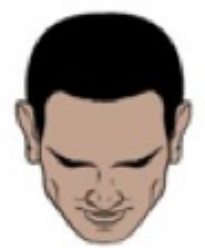

4

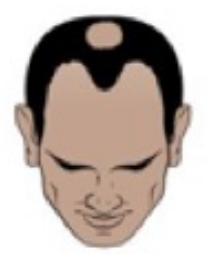

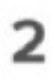

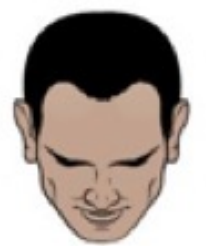

5

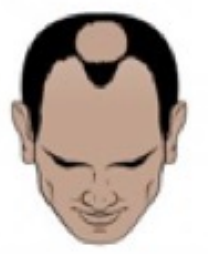

3

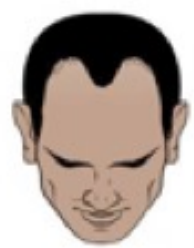

6

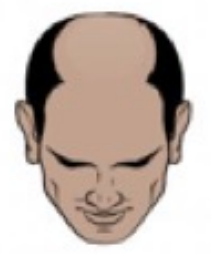

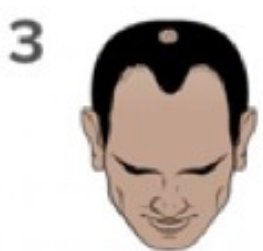

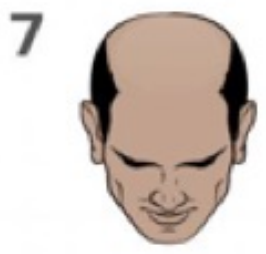




\section{Confidential}

[hh1_fname] [hh1_sname]: Referring to the illustration above, please select a number from 1-7 that best describes your hair

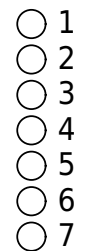

MedicationSome medicines may affect your risk of getting respiratory infections and may either protect against severe diseases or possibly increase the risk. We want to find out more about this

[hh1_fname] [hh1_sname] : Do you take any medication? $\bigcirc$ Yes

No

[hh1_fname] [hh1_sname]: Are you currently receiving treatment or taking medications that may affect your immune system?

Please select all that apply $\square$ Medication following an organ transplant $\square$ Medicines such as steroid tablets that weaken the immune system

$\square$ Targeted therapy or chemotherapy for cancer treatment

$\square$ Radiotherapy for cancer treatment

$\square$ Other treatment or medication that may affect immune system

$\square$ None of these [hh1_fname] [hh1_sname]: Do you regularly take medicine to surpress gastric acid?

Please select all that apply $\square$ Ranitidine (e.g. Zantac)

$\square$ Omeprazole (e.g. Losec)

Antacids (e.g. Rennies)

None of these [hh1_fname] [hh1_sname]: Which of the following medicines do you take?

Please select all that apply
Regularly taking Aspirin

Regularly taking "NSAIDS" e.g. Ibuprofen, nurofen, diclofenic, naproxen.

$\square$ Regularly taking blood pressure medicines ending in "-pril" such as enalapril, lisinopril, captopril, ramipril

$\square$ Regularly taking blood pressure measurements ending in "-sartan" such as losartan, valsartan, irbesartan

$\square$ Regularly taking anticoagulants e.g warfarin, ivaroxaban (Xarelto), dabigatran (Pradaxa), apixaban (Eliquis), edoxaban (Lixiana)

$\square$ Steroid tablets

$\square$ Regularly use a steroid inhaler

$\square$ Regularly take statins e.g. atorvastatin (Lipitor)

$\square$ None of these

[hh1_fname] [hh1_sname]: Which of the following medicines do you take?

Please select all that apply

\section{Steroid tablets}

Regularly use a steroid inhaler

[hh1_fname] [hh1_sname]: Do you take any vitamin supplements?

Please select all that apply

$\square$ Vitamin C Supplements

$\square$ Vitamin D Supplements

$\square$ Other

None

[hh1_fname] [hh1_sname]: Have you ever had a flu vaccine?

Yes

No 


\section{Confidential}

[hh1_fname] [hh1_sname]: Approximately when did you

have your most recent flu vaccine?

If you do not remember the exact date, please select an approximate date

Drinking and Smoking

Drinking and smoking affects the risk of many diseases. We want to find out if it affects the risk of COVID-19 infection

$\begin{array}{ll}\text { [hh1_fname] [hh1_sname]: Have you ever smoked } & \bigcirc \text { Yes } \\ \text { cigarettes regularly? } & \bigcirc \text { No }\end{array}$

cigarettes regularly?

No

[hh1_fname] [hh1_sname]: And do you smoke cigarettes $\bigcirc$ Yes

at all nowadays? $\quad O$ No

[hh1_fname] [hh1_sname]: How many cigarettes do you

smoke daily?

Please enter digits only, e.g. '5' and not 'five'

[hh1_fname] [hh1_sname]: When did you give up smoking?

Less than 3 months ago

3 - 6 months ago

More than 6 months ago but less than 1 year ago

1 year or more ago [hh1_fname] [hh1_sname]: Thinking about the past month, how often did you have a drink containing alcohol?
Daily

4-6 Times per week

2-3 Times per week

Weekly or Less

2-4 times per month

Never

[hh1_fname] [hh1_sname]: How many drinks do you have on a typical day when you are drinking?
1-2 Drinks
3-4 Drinks
5-6 Drinks
7-9 Drinks
$10+$

Accessing Health care during the lockdown

[hh1_fname] [hh1_sname]: Have you had any healthcare appointments cancelled, postponed or changed to a

Y Yes

telephone or online (including video) consultation

$\bigcirc$ No

since the start of the pandemic? 


\section{Confidential}

Please tick all that applied 


\section{Confidential}

\section{Employment status for [hh1_fname] [hh1_sname]}

Many people's work has been affected by the coronavirus and people's work can affect their chance of catching coronavirus. We'd like to know about your work and how it has been effected by the coronavirus.

[hh1 fname] [hh1 sname]: Thinking back to earlier this year, before the outbreak of the coronavirus pandemic. Which of these description best describes your work status?

\author{
Employed full time \\ Employed part time \\ Self employed full time \\ Self employed part time \\ Retired \\ Student \\ Looking after house/family (not looking for work) \\ $\bigcirc$ Permanently sick or disabled \\ $\bigcirc$ Unemployed \\ None of the above
}

[hh1 fname] [hh1_sname]: What is/was the name of your job?

[hh1_fname] [hh1_sname]: Please describe what you do/did at work

[hh1_fname] [hh1_sname]: Are you a health or social

$\bigcirc$ Yes care worker?

No

What setting do you work in?

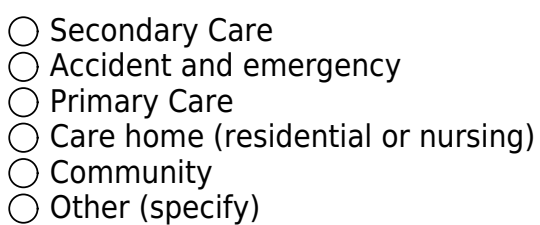

Other (Please Specify)

[hh1_fname] [hh1_sname]: Please select your healthcare profession

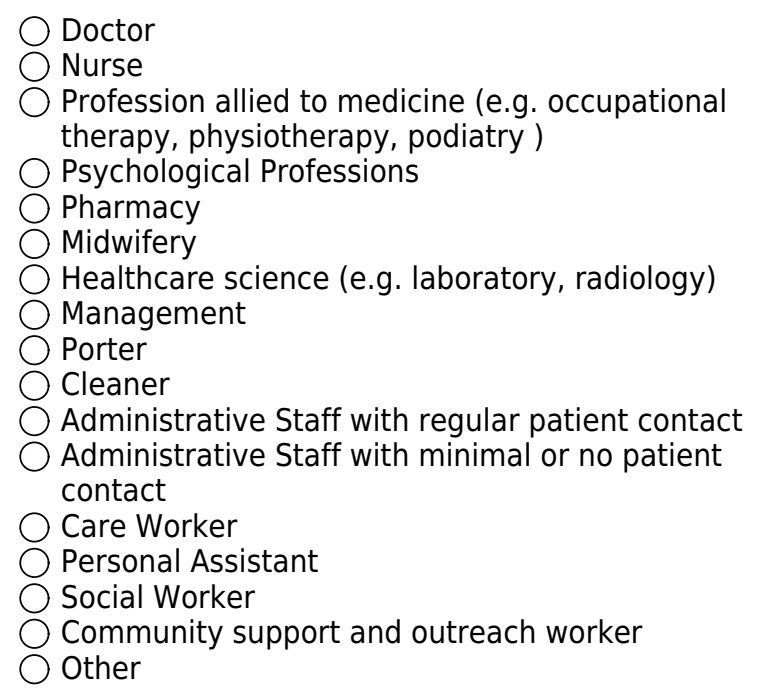

[hh1_fname] [hh1_sname]: Please specify 


\section{Confidential}

[hh1_fname] [hh1_sname]: What was your last job when you were working?

[hh1_fname] [hh1_sname]: Are you a healthcare student? e.g. medical student, student nurse

\section{Employment during the pandemic}

[hh1_fname] [hh1_sname]: BEFORE THE PANDEMIC, how many

hours of paid work did you work per week?

Please enter digits only, e.g. '5' and not 'five'

[hh1_fname] [hh1_sname]: SINCE THE PANDEMIC, during

the months of March, April, May, how many hours of

paid work have you worked since each week?

Please enter digits only, e.g. '5' and not 'five'

It looks like you have reduced your paid work during the lockdown.

[hh1_fname] [hh1_sname]: Why have you had to reduce the number of hours? $\bigcirc$ Laid off by employer with certain recall date

$\bigcirc$ Laid off or made redundant by employer with some prospect of recall

Employer cut hours

Have been put on furlough or paid leave

Using annual leave

On paid or statutory sick leave

On unpaid sick leave

Caring for children or others

Other reasons [hh1_fname] [hh1_sname] : Why have you had to reduce the number of hours?
My business has been directly affected by regulations on opening or other new regulations

My business has been directly affected by limited supplies that I need for my business

My business has been directly affected by reduced demand for my services or products

Illness

Self-isolating

Caring for children or others

Other reasons

[hh1_fname] [hh1_sname]: Have you received a written letter from your employer informing you that you have been furloughed under the Coronavirus Job Retention scheme?

\section{Working from home during the pandemic}

[hh1_fname] [hh1_sname]: How often did you WORK FROM HOME during the following three time periods:

Before the lockdown (before March 2020)

\section{(Nearly) Always}

O

\section{Often}

$O$

Sometimes

0
(Almost) Never

O 


\section{Confidential}

During the main lockdown (Mid

March to Mid May) when we

were asked to stay at Home,

Save Lives, Support the NHS

After the easing of restrictions in mid-May, when we were encouraged to go back to work if we could not work from home (Stay Alert, Control the Virus, Save Lives)

[hh1_fname] [hh1_sname]: How do/did you usually travel to work?

Please choose one or more to represent a typical day

\begin{tabular}{|c|c|c|}
\hline $\begin{array}{l}\text { Before the lockdown (before } \\
\text { March 2020) }\end{array}$ & $\begin{array}{l}\text { During the main lockdown } \\
\text { (Mid March to Mid May) when } \\
\text { we were asked to stay at } \\
\text { Home, Save Lives, Support } \\
\text { the NHS }\end{array}$ & $\begin{array}{l}\text { After the easing of } \\
\text { restrictions in mid-May, when } \\
\text { we were encouraged to go } \\
\text { back to work if we could not } \\
\text { work from home (Stay Alert, } \\
\text { Control the Virus, Save Lives) }\end{array}$ \\
\hline
\end{tabular}

By car or van

Motorcycle, moped or scooter

Taxi or minicab

Train

Underground

Bus

Tram or light railway

Cycle

Walk

Other

I did not travel to work during this period

[hh1 fname] [hh1 sname]: BEFORE LOCKDOWN, how long did

it take to get to work each day?

Please provide the time for a one-way journey in minutes or enter 0 if you did not travel to work in this period

[hh1 fname] [hh1 sname]: DURING LOCKDOWN, how long did

it take to get to work each day?

Please provide the time for a one-way journey in

minutes or enter 0 if you did not travel to work in

this period 


\section{Confidential}

[hh1_fname] [hh1_sname]: AFTER THE EASING OF

RESTRICTIONS, how long did it take to get to work each

day?

Please provide the time for a one-way journey in

minutes or enter 0 if you did not travel to work in

this period 


\section{Confidential}

\section{Finances details for [hh1_fname] [hh1_sname]}

Many people have been affected financially by the coronavirus. We'd like to know how you have been affected and how you and your household are coping.

[hh1_fname] [hh1_sname]: What is your combined household income last year?

We want to understand how COVID-19 impacts households with different levels of income
$0-9,999$
$10,000-24,999$
$25,000-49,999$
$50,000-74,999$
$75,000-99,999$
$100,000-124,999$
$125,000-149,999$
$150,000-174,999$
$175,000-199,999$
200,000 or more
Prefer not to say

[hh1_fname] [hh1_sname] : BEFORE THE PANDEMIC, how would you say you were managing financially? Would you say you were:
Living comfortably
$\bigcirc$ Doing alright
Just about getting by
Finding it quite difficult
Finding it very difficult

[hh1_fname] [hh1_sname]: How would you say you are manāging financially now? Would you say you were: [hh1_fname] [hh1_sname]: BEFORE THE PANDEMIC, have you ever needed to use a food bank?
Living comfortably

Doing alright

Just about getting by

Finding it quite difficult

Finding it very difficult [hh1_fname] [hh1_sname]: SINCE THE PANDEMIC, have you needed to use a food bank?
Never

Less than once a week

Once a week or more
Never

Less than once a week

Once a week or more 


\section{Confidential}

\section{Caring information during the lockdown - [hh1_fname] [hh1_sname]}

[hh1_fname] [hh1_sname]: About how many hours a week did you spend on childcare or home-schooling during the lockdown?

Please enter digits only, e.g. '5' and not 'five'

[hh1 fname] [hh1 sname]: Who is mainly responsible for looking after the children or home schooling?
Mainly you

Mainly your husband/wife/partner

$\bigcirc$ Jointly with your husband/wife/partner

Someone else 


\title{
Confidential
}

\begin{abstract}
[hh1 fname] [hh1 sname]: Have you EVER come into contäct with anyone that was known or presumed to have COVID-19?

Close contact includes:

Physical contact with another personA five minute conversation with someone less than 2 metres awayBeing less than 2 metres away from someone for 15 minutes or more, even if you didn't talk to each otherPlease select all that apply.
\end{abstract}

Previous COVID-19 like illness for [hh1_fname] [hh1_sname]

$\square$ No
$\square$ Yes (a household member)
$\square$ Yes (at work)
$\square$ Yes (a non-household friend or relation)
$\square$ Yes (in public)
$\square$ Yes (other)
$\square$ Don't know

Did this person have COVID confirmed by a laboratory test?
OYes
No
Don't know

\begin{tabular}{ll}
\hline [hh1_fname] [hh1_sname]: Have you had an illness & Y Yes \\
involving Cough, or Fever, or Loss of sense of smell & O No
\end{tabular}
since the 1st of January 2020 ?

[hh1 fname] [hh1 sname]: Which month did the illness start and which symptoms did you have? If you had more than one illness, you may select multiple start months

\begin{tabular}{lcccccccccccccc}
\hline & Jan & Feb & Mar & Apr & May & Jun & Jul & Aug & Sep & Oct & Nov & Dec & Jan & Feb \\
& 2020 & 2020 & 2020 & 2020 & 2020 & 2020 & 2020 & 2020 & 2020 & 2020 & 2020 & 2020 & 2021 & 2021 \\
Cough & $\square$ & $\square$ & $\square$ & $\square$ & $\square$ & $\square$ & $\square$ & $\square$ & $\square$ & $\square$ & $\square$ & $\square$ & $\square$ & $\square$ \\
Fever & $\square$ & $\square$ & $\square$ & $\square$ & $\square$ & $\square$ & $\square$ & $\square$ & $\square$ & $\square$ & $\square$ & $\square$ & $\square$ & $\square$ \\
Loss of sense of smell & $\square$ & $\square$ & $\square$ & $\square$ & $\square$ & $\square$ & $\square$ & $\square$ & $\square$ & $\square$ & $\square$ & $\square$ & $\square$ & $\square$
\end{tabular}

[hh1_fname] [hh1_sname]: CoVID-19 can cause a wide range of symptoms. Have you had an illness that you OYes think might have been COVID-19, or which was confirmed No as COVID-19?

Please describe in your own words how did the illness started and what you did when you started to feel ill?

Please describe in your own words, what symptoms developed and what did you do?

Please describe in your own words, how long did your symptoms last and are any symptoms still there?

[hh1_fname] [hh1_sname]: Have you ever had a nose or throat swab test for COVID-19?
OYes
No
Not Sure

What month was the swab taken and what was the result?

Please select all the months in which you took each test (if you took multiple) and their results 


\section{Confidential}

\begin{tabular}{|c|c|c|c|c|}
\hline & & & & Page 21 \\
\hline $\operatorname{Jan} 2020$ & The test was positive & $\begin{array}{c}\text { The test was negative } \\
\end{array}$ & $\begin{array}{c}\text { The test was unclear } \\
\bigcirc\end{array}$ & $\begin{array}{l}\text { I haven't had the } \\
\text { reøblt }\end{array}$ \\
\hline Feb 2020 & $\bigcirc$ & 0 & 0 & $\bigcirc$ \\
\hline Mar 2020 & 0 & 0 & 0 & 0 \\
\hline Apr 2020 & $\bigcirc$ & 0 & 0 & 0 \\
\hline May 2020 & $\bigcirc$ & 0 & 0 & $\bigcirc$ \\
\hline Jun 2020 & 0 & $\bigcirc$ & $\bigcirc$ & 0 \\
\hline Jul 2020 & $\bigcirc$ & $\bigcirc$ & 0 & $\bigcirc$ \\
\hline Aug 2020 & $\bigcirc$ & $\bigcirc$ & $\bigcirc$ & 0 \\
\hline Sep 2020 & 0 & 0 & 0 & 0 \\
\hline Oct 2020 & $\bigcirc$ & 0 & $\bigcirc$ & 0 \\
\hline Nov 2020 & 0 & 0 & 0 & $\bigcirc$ \\
\hline Dec 2020 & 0 & $\bigcirc$ & $\bigcirc$ & 0 \\
\hline Jan 2021 & 0 & $\bigcirc$ & 0 & $\bigcirc$ \\
\hline Feb 2021 & 0 & 0 & 0 & $\bigcirc$ \\
\hline
\end{tabular}




\section{Confidential}

\section{Thank You}

We want to know what questions you think are important so we can try to answer them with the help of tens of thousands of Virus Watch participants. Each month we will ask our participants to say what questions they would like to see answered.

[hh1_fname] [hh1_sname]: What questions would you like

Virus Watch to answer?

As we come out of a very difficult and tragic period we want to know about your three main worries related to COVID-19 and the COVID-19 response.

[hh1_fname] [hh1_sname]: What are your three main worries about the COVID-19 pandemic?

1st most worrying aspect:

2nd most worrying aspect:

3rd most worrying aspect:

Please click submit to continue 


\title{
Confidential
}

Weekly Survey

\begin{abstract}
Welcome to the weekly followup survey
surveydate

In Virus Watch we are interested in the following types of symptoms:

General symptoms (e.g. fevers, general muscle aches, headache, joint pain,extreme tiredness, trouble with daily activities around the house)Respiratory Symptoms (e.g. cough, shortness of breath, earache, sore throat, runny nose, blocked nose, sneezing, wheeze, loss or altered senses of smell or taste).Eyes (e.g. eye redness, eye pain,sticky eye, deterioration of eyesight)RashesDigestive symptoms (e.g. diarrhoea or loose stools, vomiting, nausea, abdominal pain)This survey is about symptoms in the week (Monday to Sunday) before you received the email with the survey link.
\end{abstract}

Download Symptom Diary

[Attachment: "Virus_Watch_Symptom_Diary.pdf"]

Have you or anyone in the household had any of these

symptoms in the past week?

Y Yes
No

Please continue to report weekly symptoms even if you

believe them to be related to a recent vaccine you

have had.

Did any household members receive a COVID-19 test

result in the past week?

OYes

No

Has anyone in the household been advised to

self-isolate in the past week?

Y Yes
No

Please indicate who received a result for a COVID-19 (swab or lateral flow) test in the past week: If you have had multiple tests this week please report any positive test dates OR your last negative test dates (if all were negative)

[go_arm_1][hh1_fname] [go_arm_1][hh1_sname]

[go_arm_1][hh1_fname] [go_arm_1][hh1_sname]: What was the result of the COVID-19 test?

Positive

Negative

Unclear

[go_arm_1][hh1_fname] [go_arm_1][hh1_sname]: When was the test taken?

[go_arm_1][hh1_fname] [go_arm_1][hh1_sname]: When did you receive the test result?

[go_arm_1][hh2_fname] [go_arm_1][hh2_sname]

[go_arm_1][hh2_fname] [go_arm_1][hh2_sname]: What was the result of the COVID-19 test?
Positive
Negative
Unclear

[go_arm_1][hh2_fname] [go_arm_1][hh2_sname]: When was the test taken? 


\section{Confidential}

[go_arm_1][hh2_fname] [go_arm_1][hh2_sname]: When did you receive the test result?

[go_arm_1][hh3_fname] [go_arm_1][hh3_sname]

[go_arm_1][hh3_fname] [go_arm_1][hh3_sname]: What was the result of the COVID-19 test?

Positive

Negative

Unclear

[go_arm_1][hh3_fname] [go_arm_1][hh3_sname]: When was the test taken?

[go_arm_1][hh3 fname] [go_arm_1][hh3_sname]: When did you receive the test result?

[go_arm_1][hh4_fname] [go_arm_1][hh4_sname]

[go_arm_1][hh4_fname] [go_arm_1][hh4_sname]: What was the result of the COVID-19 test?
Positive
$\bigcirc$ Negative
$\bigcirc$ Unclear

[go_arm_1][hh4_fname] [go_arm_1][hh4_sname]: When was the test taken?

[go_arm_1][hh4_fname] [go_arm_1][hh4_sname]: When did you receive the test result?

[go_arm_1][hh5_fname] [go_arm_1][hh5_sname]

[go_arm_1][hh5_fname] [go_arm_1][hh5_sname]: What was the result of the COVID-19 test?

\section{Positive \\ Negative \\ Unclear}

[go_arm_1][hh5_fname] [go_arm_1][hh5_sname]: When was the test taken?

[go_arm_1][hh5_fname] [go_arm_1][hh5_sname]: When did you receive the test result?

[go_arm_1][hh6_fname] [go_arm_1][hh6_sname]

[go_arm_1][hh6_fname] [go_arm_1][hh6_sname]: What was the result of the COVID-19 test?

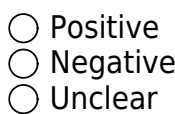

Negative

[go_arm_1][hh6_fname] [go_arm_1][hh6_sname]: When was the test taken?

[go_arm_1][hh6_fname] [go_arm_1][hh6_sname]: When did you receive the test result?

Please indicate who was advised to self isolate in the past week:

[go_arm_1][hh1_fname] [go_arm_1][hh1_sname] 


\section{Confidential}

[go_arm_1][hh1_fname] [go_arm_1][hh1_sname]: Which of the following led to the request to self-isolate?
The Test and Trace programme advised me to self-isolate because I have symptoms of COVID-19

The Test and Trace programme advised me to self-isolate because I am had a positive test result

The Test and Trace programme advised me to self isolate because I was in contact with a COVID-19 case

The NHS COVID-19 app alerted me that I had been in contact with a COVID-19 case

I have returned from a country where quarantine is advised after return.

Other reason

[go_arm_1][hh2_fname] [go_arm_1][hh2_sname]

[go_arm_1][hh2_fname] [go_arm_1][hh2_sname]: Which of the following led to the request to self-isolate?
The Test and Trace programme advised me to self-isolate because I have symptoms of COVID-19

$\bigcirc$ The Test and Trace programme advised me to self-isolate because I am had a positive test result

The Test and Trace programme advised me to self isolate because I was in contact with a COVID-19 case

The NHS COVID-19 app alerted me that I had been in contact with a COVID-19 case

I have returned from a country where quarantine is advised after return.

$\bigcirc$ Other reason

[go_arm_1][hh3_fname] [go_arm_1][hh3_sname]

[go_arm_1][hh3_fname] [go_arm_1][hh3_sname]: Which of the following led to the request to self-isolate?
The Test and Trace programme advised me to self-isolate because I have symptoms of COVID-19

$\bigcirc$ The Test and Trace programme advised me to self-isolate because I am had a positive test result

The Test and Trace programme advised me to self isolate because I was in contact with a COVID-19 case

The NHS COVID-19 app alerted me that I had been in contact with a COVID-19 case

$\bigcirc$ I have returned from a country where quarantine is advised after return.

Other reason

[go_arm_1][hh4_fname] [go_arm_1][hh4_sname] 


\section{Confidential}

[go_arm_1][hh4_fname] [go_arm_1][hh4_sname]: Which of the following led to the request to self-isolate?
The Test and Trace programme advised me to self-isolate because I have symptoms of COVID-19

The Test and Trace programme advised me to self-isolate because I am had a positive test result

The Test and Trace programme advised me to self isolate because I was in contact with a COVID-19 case

The NHS COVID-19 app alerted me that I had been in contact with a COVID-19 case

I have returned from a country where quarantine is advised after return.

Other reason

[go_arm_1][hh5_fname] [go_arm_1][hh5_sname]

[go_arm_1][hh5_fname] [go_arm_1][hh5_sname]: Which of the following led to the request to self-isolate?
The Test and Trace programme advised me to self-isolate because I have symptoms of COVID-19

$\bigcirc$ The Test and Trace programme advised me to self-isolate because I am had a positive test result

The Test and Trace programme advised me to self isolate because I was in contact with a COVID-19 case

The NHS COVID-19 app alerted me that I had been in contact with a COVID-19 case

I have returned from a country where quarantine is advised after return.

$\bigcirc$ Other reason

[go_arm_1][hh6_fname] [go_arm_1][hh6_sname]

[go_arm_1][hh6_fname] [go_arm_1][hh6_sname]: Which of the following led to the request to self-isolate?
The Test and Trace programme advised me to self-isolate because I have symptoms of COVID-19

$\bigcirc$ The Test and Trace programme advised me to self-isolate because I am had a positive test result

$\bigcirc$ The Test and Trace programme advised me to self isolate because I was in contact with a COVID-19 case

The NHS COVID-19 app alerted me that I had been in contact with a COVID-19 case

I have returned from a country where quarantine is advised after return.

Other reason

Please indicate who has had symptoms in the past week:

[go_arm_1][hh1_fname] [go_arm_1][hh1_sname]:
Yes, symptoms that have been present for less than 2 months

Yes, symptoms that developed after a COVID-19 like illness and have lasted for more than 2 months

$\bigcirc$ Yes, symptoms that are part of a long term chronic illness

No symptoms 


\section{Confidential}

[go_arm_1][hh2_fname] [go_arm_1][hh2_sname]:
Yes, symptoms that have been present for less than 2 months

$\bigcirc$ Yes, symptoms that developed after a COVID-19 like illness and have lasted for more than 2 months

$\bigcirc$ Yes, symptoms that are part of a long term chronic illness

No symptoms $\bigcirc$ Yes, symptoms that have been present for less than 2 months

$\bigcirc$ Yes, symptoms that developed after a COVID-19 like illness and have lasted for more than 2 months

$\bigcirc$ Yes, symptoms that are part of a long term chronic illness

No symptoms
Yes, symptoms that have been present for less than 2 months

Yes, symptoms that developed after a COVID-19 like illness and have lasted for more than 2 months

$\bigcirc$ Yes, symptoms that are part of a long term chronic illness

No symptoms

[go_arm_1][hh5_fname] [go_arm_1][hh5_sname]:
Yes, symptoms that have been present for less than 2 months

Yes, symptoms that developed after a COVID-19 like illness and have lasted for more than 2 months

$\bigcirc$ Yes, symptoms that are part of a long term chronic illness

No symptoms
Yes, symptoms that have been present for less than 2 months

Yes, symptoms that developed after a COVID-19 like illness and have lasted for more than 2 months

Yes, symptoms that are part of a long term chronic illness

No symptoms

Please indicate whether the following members have received a result for a COVID-19 (swab or lateral flow) test in the past week: If you have had multiple tests this week please report any positive test dates OR your last negative test dates (if all were negative)

[go_arm_1][hh1_fname] [go_arm_1][hh1_sname]:

[go_arm_1][hh1_fname] [go_arm_1][hh1_sname]: What was the result of this test?

\section{Positive \\ Negative \\ Onclear}

[go_arm_1][hh1_fname] [go_arm_1][hh1_sname]: When was the test taken?

[go_arm_1][hh1_fname] [go_arm_1][hh1_sname]: When did you receive the test result?

[go_arm_1][hh2_fname] [go_arm_1][hh2_sname]: 


\section{Confidential}

[go_arm_1][hh2_fname] [go_arm_1][hh2_sname]: What was the result of this test?
Positive
Negative
Unclear

[go_arm_1][hh2_fname] [go_arm_1][hh2_sname]: When was the test taken?

[go_arm_1][hh2_fname] [go_arm_1][hh2_sname]: When did you receive the test result?

[go_arm_1][hh3_fname] [go_arm_1][hh3_sname]:

[go_arm_1][hh3_fname] [go_arm_1][hh3_sname]: What was the result of this test?

\section{Positive \\ Negative \\ $\bigcirc$ Unclear}

[go_arm_1][hh3_fname] [go_arm_1][hh3_sname]: When was the test taken?

[go_arm_1][hh3_fname] [go_arm_1][hh3_sname]: When did you receive the test result?

[go_arm_1][hh4_fname] [go_arm_1][hh4_sname]:

[go_arm_1][hh4_fname] [go_arm_1][hh4_sname]: What was the result of this test?

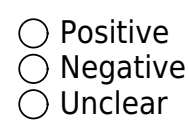

[go_arm_1][hh4_fname] [go_arm_1][hh4_sname]: When was the test taken?

[go_arm_1][hh4_fname] [go_arm_1][hh4_sname]: When did you receive the test result?

[go_arm_1][hh5_fname] [go_arm_1][hh5_sname]:

[go_arm_1][hh5_fname] [go_arm_1][hh5_sname]: What was the result of this test?

\section{Positive \\ Negative \\ Unclear}

[go_arm_1][hh5_fname] [go_arm_1][hh5_sname]: When was the test taken?

[go_arm_1][hh5_fname] [go_arm_1][hh5_sname]: When did you receive the test result?

[go_arm_1][hh6_fname] [go_arm_1][hh6_sname]:

[go_arm_1][hh6_fname] [go_arm_1][hh6_sname]: What was the result of this test?

\section{Positive \\ Negative \\ Unclear}

[go_arm_1][hh6_fname] [go_arm_1][hh6_sname]: When was the test taken? 


\section{Confidential}

[go_arm_1][hh6_fname] [go_arm_1][hh6_sname]: When did you receive the test result?

Please indiciate whether the following household members been advised to self-isolate in the past week:

[go_arm_1][hh1_fname] [go_arm_1][hh1_sname]

[go_arm_1][hh1_fname] [go_arm_1][hh1_sname]: Which of the following led to the request to self-isolate?
The Test and Trace programme advised me to self-isolate because I have symptoms of COVID-19

The Test and Trace programme advised me to self-isolate because I am had a positive test result

The Test and Trace programme advised me to self isolate because I was in contact with a COVID-19 case

The NHS COVID-19 app alerted me that I had been in contact with a COVID-19 case

I have returned from a country where quarantine is advised after return.

$\bigcirc$ Other reason

[go_arm_1][hh2_fname] [go_arm_1][hh2_sname]

[go arm 1][hh2 fname] [go arm 1][hh2 sname]: Which of the following led to the request to self-isolate?
The Test and Trace programme advised me to self-isolate because I have symptoms of COVID-19

$\bigcirc$ The Test and Trace programme advised me to self-isolate because I am had a positive test result

The Test and Trace programme advised me to self isolate because I was in contact with a COVID-19 case

The NHS COVID-19 app alerted me that I had been in contact with a COVID-19 case

I have returned from a country where quarantine is advised after return.

Other reason

[go_arm_1][hh3_fname] [go_arm_1][hh3_sname]

[go_arm_1][hh3_fname] [go_arm_1][hh3_sname]: Which of the following led to the request to self-isolate?
The Test and Trace programme advised me to self-isolate because I have symptoms of COVID-19

$\bigcirc$ The Test and Trace programme advised me to self-isolate because I am had a positive test result

The Test and Trace programme advised me to self isolate because I was in contact with a COVID-19 case

The NHS COVID-19 app alerted me that I had been in contact with a COVID-19 case

I have returned from a country where quarantine is advised after return.

Other reason

[go_arm_1][hh4_fname] [go_arm_1][hh4_sname] 


\section{Confidential}

[go_arm_1][hh4_fname] [go_arm_1][hh4_sname]: Which of the following led to the request to self-isolate?
The Test and Trace programme advised me to self-isolate because I have symptoms of COVID-19

The Test and Trace programme advised me to self-isolate because I am had a positive test result

The Test and Trace programme advised me to self isolate because I was in contact with a COVID-19 case

The NHS COVID-19 app alerted me that I had been in contact with a COVID-19 case

I have returned from a country where quarantine is advised after return.

Other reason

[go_arm_1][hh5_fname] [go_arm_1][hh5_sname]

[go_arm_1][hh5_fname] [go_arm_1][hh5_sname]: Which of the following led to the request to self-isolate?
The Test and Trace programme advised me to self-isolate because I have symptoms of COVID-19

$\bigcirc$ The Test and Trace programme advised me to self-isolate because I am had a positive test result

The Test and Trace programme advised me to self isolate because I was in contact with a COVID-19 case

The NHS COVID-19 app alerted me that I had been in contact with a COVID-19 case

I have returned from a country where quarantine is advised after return.

$\bigcirc$ Other reason

[go_arm_1][hh6_fname] [go_arm_1][hh6_sname]

[go arm 1][hh6 fname] [go arm 1][hh6 sname]: Which of the following led to the request to self-isolate?
The Test and Trace programme advised me to self-isolate because I have symptoms of COVID-19

The Test and Trace programme advised me to self-isolate because I am had a positive test result

The Test and Trace programme advised me to self isolate because I was in contact with a COVID-19 case

The NHS COVID-19 app alerted me that I had been in contact with a COVID-19 case

$\bigcirc$ I have returned from a country where quarantine is advised after return.

Other reason 


\section{Confidential}

\section{Vaccination}

Has anyone in the household received a COVID-19

vaccine in the past week?

OYes

O No

Unsure (e.g. as part of a blinded COVID-19 trial)

Please indicate who received a COVID-19 vaccine:

[go_arm_1][hh1_fname] [go_arm_1][hh1_sname]

[go_arm_1][hh1_fname] [go_arm_1][hh1_sname]: Please select which dose(s) of the $\bar{C} O V I \bar{D}-19$ vaccine you

$\square$ 1st Dose received?

2nd Dose

[go_arm_1][hh1_fname] [go_arm_1][hh1_sname]: Which type of vaccine did you receive as the 1st dose?
Pfizer Biontech vaccine
Oxford AstraZeneca vaccine
Other vaccine
$\bigcirc$ Don't know/Don't remember

[go arm 1][hh1 fname] [go arm_1][hh1_sname]: What date did you receive the 1st dose?

(dd-mm-yyyy)

Please provide an estimate if you cannot recall the date

[go_arm_1][hh1_fname] [go_arm_1][hh1_sname]: Which type of vaccine did you receive as the 2 nd dose?
Pfizer Biontech vaccine
Oxford AstraZeneca vaccine
Other vaccine
Don't know/Don't remember

[go_arm_1][hh1_fname] [go_arm_1][hh1_sname]: What date
did you receive the 2 nd dose?

(dd-mm-yyyy)

Please provide an estimate if you cannot recall the

date

[go_arm_1][hh2_fname] [go_arm_1][hh2_sname]

[go_arm_1][hh2_fname] [go_arm_1][hh2_sname]: Please selēct which dose(s) of the C̄OVID-19 vačcine you received?

1st Dose

2nd Dose [go arm_1][hh2 fname] [go arm 1][hh2 sname]: Which type of vaccine $\overline{\text { did }}$ you receive as the 1 st dose?
Pfizer Biontech vaccine

Oxford AstraZeneca vaccine

Other vaccine

Don't know/Don't remember

[go_arm_1][hh2_fname] [go_arm_1][hh2_sname]: What date did you receive the 1st dose? (dd-mm-yyyy)

Please provide an estimate if you cannot recall the date 


\section{Confidential}

[go_arm_1][hh2_fname] [go_arm_1][hh2_sname]: Which type of vaccine $\overline{d i d}$ you receive as the 2 nd dose?
Pfizer Biontech vaccine

Oxford AstraZeneca vaccine

Other vaccine

Don't know/Don't remember

[go arm_1][hh2 fname] [go arm 1][hh2 sname]: What date

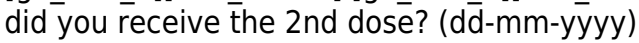

Please provide an estimate if you cannot recall the date

[go_arm_1][hh3_fname] [go_arm_1][hh3_sname]

[go_arm_1][hh3 fname] [go arm_1][hh3_sname]: Please

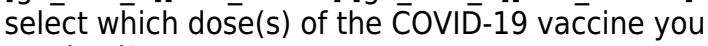
received?

$\square$ 1st Dose

$\square$ 2nd Dose

[go_arm_1][hh3 fname] [go_arm_1][hh3_sname]: Which type of vaccine did you receive as the 1st dose?
Pfizer Biontech vaccine
Oxford AstraZeneca vaccine
Other vaccine
Don't know/Don't remember

[go_arm_1][hh3_fname] [go_arm_1][hh3_sname]: What date did you receive the 1 st dose? (dd-mm-yyyy)

Please provide an estimate if you cannot recall the date

[go_arm_1][hh3_fname] [go_arm_1][hh3_sname]: Which type of vaccine did you receive as the 2 nd dose?
Pfizer Biontech vaccine
Oxford AstraZeneca vaccine
Other vaccine
Don't know/Don't remember

[go_arm_1][hh3_fname] [go_arm_1][hh3_sname]: What date did you receive the 2 nd dose? (d⿳亠口冋-mm-yyyy)

Please provide an estimate if you cannot recall the date

[go_arm_1][hh4_fname] [go_arm_1][hh4_sname]

[go arm 1][hh4 fname] [go arm 1][hh4 sname]: Please select which dose(s) of the $\bar{C} O V I \bar{D}-19$ vaccine you received?

$\square$ 1st Dose
$\square$ 2nd Dose

[go_arm_1][hh4_fname] [go_arm_1][hh4_sname]: Which type of vaccine did you receive as the $1 \overline{s t}$ dose?
Pfizer Biontech vaccine

Oxford AstraZeneca vaccine

Other vaccine

$\bigcirc$ Don't know/Don't remember

[go_arm_1][hh4_fname] [go_arm_1][hh4_sname]: What date did you receive the 1st dose? (dd-mm-yyyy)

Please provide an estimate if you cannot recall the date 


\section{Confidential}

[go_arm_1][hh4_fname] [go_arm_1][hh4_sname]: Which type of vaccine $\overline{d i d}$ you receive as the 2 nd dose?
Pfizer Biontech vaccine

Oxford AstraZeneca vaccine

Other vaccine

Don't know/Don't remember

[go arm_1][hh4 fname] [go arm 1][hh4_sname]: What date

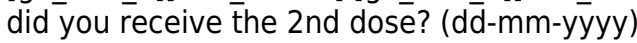

Please provide an estimate if you cannot recall the date

[go_arm_1][hh5_fname] [go_arm_1][hh5_sname]

[go_arm_1][hh5 fname] [go arm_1][hh5_sname]: Please

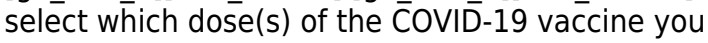
received?

$\square$ 1st Dose

$\square$ 2nd Dose

[go_arm_1][hh5_fname] [go_arm_1][hh5_sname]: Which type of vaccine did you receive as the 1st dose?
Pfizer Biontech vaccine
Oxford AstraZeneca vaccine
Other vaccine
Don't know/Don't remember

[go_arm_1][hh5_fname] [go_arm_1][hh5_sname]: What date did you receive the 1st dose? (dd-mm-yyyy)

Please provide an estimate if you cannot recall the date

[go_arm_1][hh5_fname] [go_arm_1][hh5_sname]: Which type of vaccine did you receive as the 2 nd dose?
Pfizer Biontech vaccine
Oxford AstraZeneca vaccine
Other vaccine
Don't know/Don't remember

[go_arm_1][hh5_fname] [go_arm_1][hh5_sname]: What date did you receive the 2 nd dose? (d⿳亠口冋-mm-yyyy)

Please provide an estimate if you cannot recall the date

[go_arm_1][hh6_fname] [go_arm_1][hh6_sname]

[go arm 1][hh6 fname] [go arm 1][hh6 sname]: Please select which dose(s) of the $\bar{C} O V I \bar{D}-19$ vaccine you received?

$\square$ 1st Dose

[go_arm_1][hh6_fname] [go_arm_1][hh6_sname]: Which type of vaccine did you receive as the 1st dose?
Pfizer Biontech vaccine
Oxford AstraZeneca vaccine
Other vaccine
$\bigcirc$ Don't know/Don't remember

[go_arm_1][hh6_fname] [go_arm_1][hh6_sname]: What date did you receive the 1st dose? (dd-mm-yyyy)

Please provide an estimate if you cannot recall the date 


\section{Confidential}

[go_arm_1][hh6_fname] [go_arm_1][hh6_sname]: Which type of vaccine did you receive as the 2 nd dose?
Pfizer Biontech vaccine

Oxford AstraZeneca vaccine

Other vaccine

Don't know/Don't remember

[go_arm_1][hh6_fname] [go_arm_1][hh6_sname]: What date

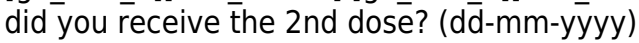

Please provide an estimate if you cannot recall the date

Thank you for letting us know that someone in your household has been ill. We hope they feel better soon. Please always follow NHS and Public Health advice when someone is ill. We will always have a link to the latest COVID-19 advice on the Virus Watch Website.

If anyone has new symptoms to report, the following survey will ask about any illness and related health care as well as asking about isolation, time off work, measures to help stop infections spreading and activities that household members have done in the last week.

The survey usually takes about 10 minutes to complete for each member of the household who has been ill.

Thank you for completing the survey - we will be in touch again next week. 


\section{Confidential}

\section{Wkhh1}

\section{Symptoms - [go_arm_1][hh1_fname] [go_arm_1][hh1_sname]}

The following sections are about the symptoms, use of treatments and access of medical services in the past week (the Monday-Sunday before you received the email with this survey link).

[go_arm_1][hh1_fname]: What parts of the body did your symptoms affect?

Select all that apply $\square$ General symptoms (fevers, general muscle aches, headache, joint pain,extreme tiredness, trouble with daily activities around the house)

$\square$ Respiratory Symptoms (e.g. cough, shortness of breath, earache, sore throat, runny nose, blocked nose, sneezing, wheeze, loss or altered senses of smell or taste)

$\square$ Eyes (e.g. eye redness, eye pain, sticky eye, deterioration of eyesight)

$\square$ Rash

$\square$ Digestive symptoms (e.g. diarrhoea or loose stools, vomiting, nausea, abdominal pain) [go_arm_1][hh1_fname] [go_arm_1][hh1_sname]: Which days did you have symptoms?

Please check all days that you had any of the above symptoms.

$\square$ Monday
$\square$ Tuesday
$\square$ Wednesday
$\square$ Thursday
$\square$ Friday
$\square$ Saturday
$\square$ Sunday




\section{Confidential}

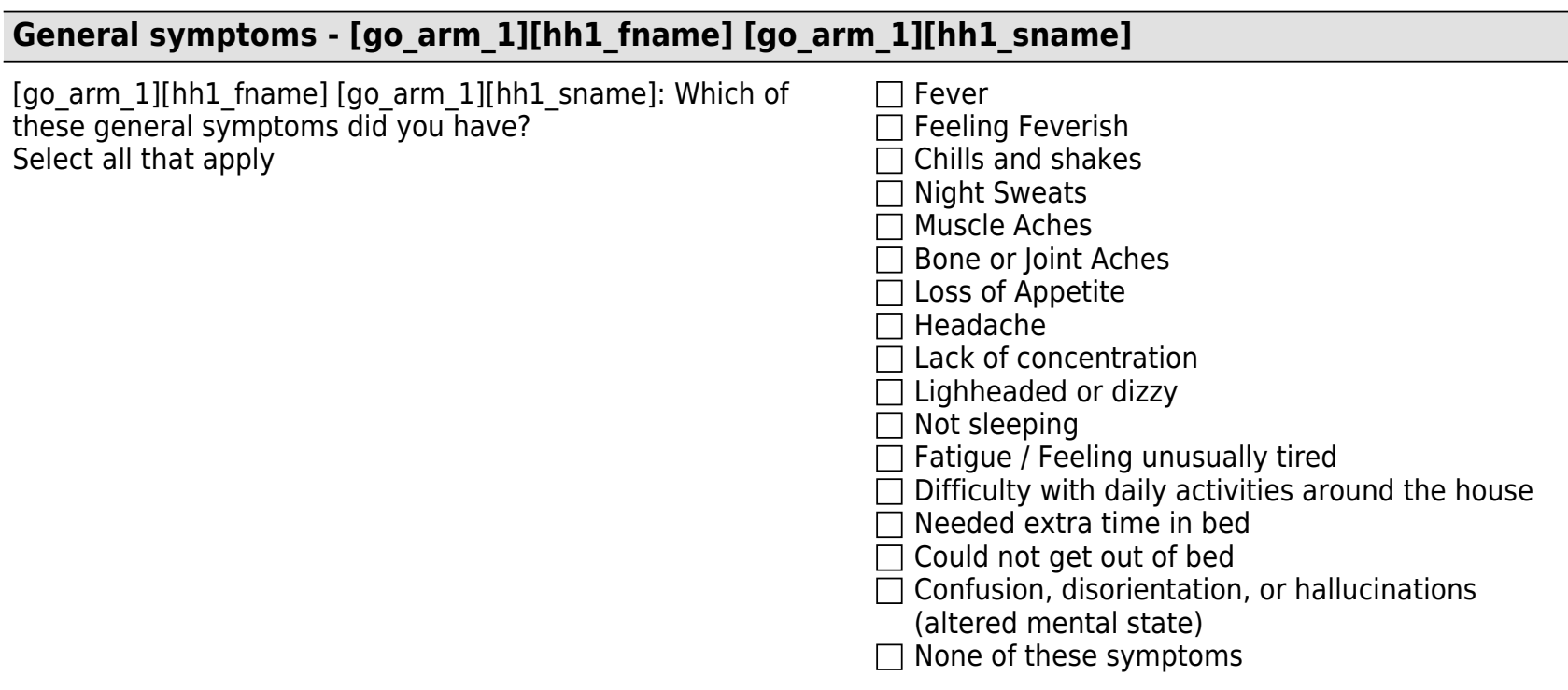

Please identify how severe your symptoms were

Fever

$\begin{array}{ccccc}\text { Less than 37.8 C } & 37.8-38.9 \mathrm{C} & 39-39.9 \mathrm{C} & 40 \mathrm{C}(104 \mathrm{~F}) \text { or } & \text { Did not take } \\ (100.0 \mathrm{~F}) & (100-102 \mathrm{~F}) & (102-103.9 \mathrm{~F}) & \text { more } & \text { temperature } \\ & & & & \text { Don't remember }\end{array}$

\begin{tabular}{|c|c|c|c|c|}
\hline Monday & $\bigcirc$ & $\bigcirc$ & 0 & $\bigcirc$ \\
\hline Tuesday & $\bigcirc$ & $\bigcirc$ & $\bigcirc$ & $\bigcirc$ \\
\hline Wednesday & $\bigcirc$ & 0 & $\bigcirc$ & $\bigcirc$ \\
\hline Thursday & $\bigcirc$ & 0 & $\bigcirc$ & $\bigcirc$ \\
\hline Friday & $\bigcirc$ & $\bigcirc$ & $\bigcirc$ & $\bigcirc$ \\
\hline Saturday & $\bigcirc$ & 0 & $\bigcirc$ & $\bigcirc$ \\
\hline Sunday & 0 & $\Omega$ & $\Omega$ & $\cap$ \\
\hline
\end{tabular}

\section{Feeling Feverish}

\begin{tabular}{|c|c|c|c|c|}
\hline & None & Mild & Moderate & Severe \\
\hline Monday & $\bigcirc$ & 0 & $\bigcirc$ & $\bigcirc$ \\
\hline Tuesday & $\bigcirc$ & $\bigcirc$ & $\bigcirc$ & $\bigcirc$ \\
\hline Wednesday & $\bigcirc$ & $\bigcirc$ & $\bigcirc$ & $\bigcirc$ \\
\hline Thursday & $\bigcirc$ & $\bigcirc$ & $\bigcirc$ & $\bigcirc$ \\
\hline Friday & 0 & $\bigcirc$ & $\bigcirc$ & $\bigcirc$ \\
\hline Saturday & $\bigcirc$ & $\bigcirc$ & $\bigcirc$ & $\bigcirc$ \\
\hline Sunday & 0 & 0 & 0 & 0 \\
\hline
\end{tabular}

Chills and Shakes 


\section{Confidential}

\section{Night Sweats}

\begin{tabular}{|c|c|c|c|c|}
\hline & None & Mild & Moderate & Severe \\
\hline Monday & 0 & 0 & $\bigcirc$ & 0 \\
\hline Tuesday & 0 & 0 & 0 & 0 \\
\hline Wednesday & 0 & $\bigcirc$ & 0 & 0 \\
\hline Thursday & 0 & 0 & 0 & 0 \\
\hline Friday & 0 & 0 & 0 & 0 \\
\hline Saturday & 0 & 0 & 0 & 0 \\
\hline Sunday & 0 & 0 & 0 & 0 \\
\hline
\end{tabular}

Muscle Ache

\begin{tabular}{lcccc}
\hline Monday & None & Mild & Moderate & Severe \\
Tuesday & 0 & 0 & 0 & 0 \\
Wednesday & 0 & 0 & 0 & 0 \\
Thursday & 0 & 0 & 0 & 0 \\
Friday & 0 & 0 & 0 & 0 \\
Saturday & 0 & 0 & 0 & 0 \\
Sunday & 0 & 0 & 0 & 0
\end{tabular}

Bone or Joint ache

\begin{tabular}{lcccc}
\hline Monday & None & Mild & Moderate & Severe \\
Tuesday & 0 & 0 & 0 & $\bigcirc$ \\
Wednesday & 0 & 0 & 0 & 0 \\
Thursday & 0 & 0 & 0 & 0 \\
Friday & 0 & 0 & 0 & 0 \\
Saturday & 0 & 0 & 0 & 0 \\
Sunday & 0 & 0 & 0 & 0 \\
& 0 & 0 & 0 & 0 \\
\hline
\end{tabular}

Loss of Appetite 


\section{Confidential}

\section{Headache}

\begin{tabular}{lcccc}
\hline Monday & None & Mild & Moderate & Severe \\
Tuesday & 0 & 0 & 0 & 0 \\
Wednesday & 0 & 0 & 0 & 0 \\
Thursday & 0 & 0 & 0 & 0 \\
Friday & 0 & 0 & 0 & 0 \\
Saturday & 0 & 0 & 0 & $\bigcirc$ \\
Sunday & 0 & 0 & 0 & 0 \\
& 0 & 0 & 0 & \\
\hline
\end{tabular}

Confusion, disorientation, or hallucinations (altered mental state)

\begin{tabular}{lcccc}
\hline Monday & None & Mild & Moderate & Severe \\
Tuesday & 0 & 0 & 0 & 0 \\
Wednesday & 0 & 0 & 0 & 0 \\
Thursday & 0 & 0 & 0 & 0 \\
Friday & 0 & 0 & 0 & 0 \\
Saturday & 0 & 0 & 0 & 0 \\
Sunday & 0 & 0 & 0 & 0 \\
& 0 & 0 & 0 & \\
\hline Lack of Concentration & & & & 0 \\
\hline Monday & & & 0 & 0 \\
Tuesday & None & Mild & 0 & 0 \\
Wednesday & 0 & 0 & 0 & 0 \\
Thursday & 0 & 0 & 0 & 0 \\
Friday & 0 & 0 & 0 & 0 \\
Saturday & 0 & 0 & 0 & 0 \\
Sunday & 0 & 0 & 0 & \\
& 0 & 0 & & 0 \\
\hline
\end{tabular}

Lightheaded or Dizzy 


\section{Confidential}

\section{Not Sleeping}

\begin{tabular}{|c|c|c|c|c|}
\hline & None & Mild & Moderate & Severe \\
\hline Monday & 0 & 0 & $\bigcirc$ & 0 \\
\hline Tuesday & 0 & 0 & 0 & 0 \\
\hline Wednesday & 0 & $\bigcirc$ & 0 & 0 \\
\hline Thursday & 0 & 0 & 0 & 0 \\
\hline Friday & 0 & 0 & 0 & 0 \\
\hline Saturday & 0 & 0 & 0 & 0 \\
\hline Sunday & 0 & 0 & 0 & 0 \\
\hline
\end{tabular}

Fatigue/Feeling Unusually Tired

\begin{tabular}{|c|c|c|c|c|}
\hline & None & Mild & Moderate & Severe \\
\hline Monday & $\bigcirc$ & 0 & 0 & 0 \\
\hline Tuesday & 0 & 0 & 0 & 0 \\
\hline Wednesday & $\bigcirc$ & 0 & 0 & $\bigcirc$ \\
\hline Thursday & 0 & 0 & 0 & 0 \\
\hline Friday & 0 & 0 & 0 & 0 \\
\hline Saturday & $\bigcirc$ & 0 & $\bigcirc$ & 0 \\
\hline Sunday & $\bigcirc$ & 0 & 0 & 0 \\
\hline
\end{tabular}

Difficulties with Daily Activities

\begin{tabular}{lcccc}
\hline Monday & None & Mild & Moderate & Severe \\
Tuesday & 0 & 0 & 0 & $\bigcirc$ \\
Wednesday & 0 & 0 & 0 & 0 \\
Thursday & 0 & 0 & 0 & 0 \\
Friday & 0 & 0 & 0 & 0 \\
Saturday & 0 & 0 & 0 & 0 \\
Sunday & 0 & 0 & 0 & 0 \\
& 0 & 0 & 0 & 0 \\
\hline
\end{tabular}

Needed extra time in bed 


\section{Confidential}

\begin{tabular}{lcccc} 
& & & Page 6 \\
\hline Monday & None & Mild & Moderate & Severe \\
Tuesday & 0 & 0 & 0 & 0 \\
Wednesday & 0 & 0 & 0 & 0 \\
Thursday & 0 & 0 & 0 & 0 \\
Friday & 0 & 0 & 0 & 0 \\
Saturday & 0 & 0 & 0 & $\bigcirc$ \\
Sunday & 0 & 0 & 0 & 0
\end{tabular}

Could not get out of bed

\begin{tabular}{lcccc}
\hline Monday & None & Mild & Moderate & Severe \\
Tuesday & 0 & 0 & 0 & $\bigcirc$ \\
Wednesday & 0 & 0 & 0 & 0 \\
Thursday & 0 & 0 & 0 & 0 \\
Friday & 0 & 0 & 0 & 0 \\
Saturday & 0 & 0 & 0 & 0 \\
Sunday & 0 & 0 & 0 & 0 \\
Sun & 0 & 0 & 0 & 0
\end{tabular}




\section{Confidential}

\begin{tabular}{|c|c|}
\hline \multicolumn{2}{|c|}{ Respiratory Symptoms - [go_arm_1][hh1_fname] [go_arm_1][hh1_sname] } \\
\hline $\begin{array}{l}\text { [go_arm_1][hh1_fname] [go_arm_1][hh1_sname]: Which } \\
\text { Respiratory symptoms did you have? } \\
\text { Select all that apply }\end{array}$ & $\begin{array}{l}\square \text { Runny Nose } \\
\square \text { Blocked Nose } \\
\square \text { Sinus pain / congestion } \\
\square \text { Dry cough } \\
\square \text { Coughing up Green Phlegm } \\
\square \text { Coughing up White Phlegm } \\
\square \text { Loss or change to sense of smell } \\
\square \text { Loss or change to sense of taste } \\
\square \text { Sneezing } \\
\square \text { Sore Throat } \\
\square \text { Swollen tonsils } \\
\square \text { Swollen glands (enlarged lymph nodes) } \\
\square \text { Ear pain or change in hearing } \\
\square \text { Fluid leaking from ear } \\
\square \text { Shortness of breath difficulty breathing } \\
\square \text { Wheezing } \\
\square \text { Chest pain (not changed by breathing or moving) } \\
\square \text { Chest pain when breathing in } \\
\square \text { None of these symptoms }\end{array}$ \\
\hline
\end{tabular}

Please identify how severe your symptoms were

Runny Nose

\begin{tabular}{|c|c|c|c|c|}
\hline & None & Mild & Moderate & Severe \\
\hline Monday & $\bigcirc$ & $\bigcirc$ & $\bigcirc$ & $\bigcirc$ \\
\hline Tuesday & 0 & O & O & O \\
\hline Wednesday & $\bigcirc$ & $\bigcirc$ & $\bigcirc$ & $\bigcirc$ \\
\hline Thursday & $\bigcirc$ & $\bigcirc$ & $\bigcirc$ & $\bigcirc$ \\
\hline Friday & $\bigcirc$ & $\bigcirc$ & $\bigcirc$ & $\bigcirc$ \\
\hline Saturday & $\bigcirc$ & $\bigcirc$ & $\bigcirc$ & $\bigcirc$ \\
\hline Sunday & $\bigcirc$ & $\bigcirc$ & $\bigcirc$ & 0 \\
\hline
\end{tabular}

\section{Blocked Nose}

\begin{tabular}{|c|c|c|c|c|}
\hline & None & Mild & Moderate & Severe \\
\hline Monday & $\bigcirc$ & $\bigcirc$ & 0 & $\bigcirc$ \\
\hline Tuesday & $\bigcirc$ & $\bigcirc$ & $\bigcirc$ & $\bigcirc$ \\
\hline Wednesday & $\bigcirc$ & 0 & $\bigcirc$ & $\bigcirc$ \\
\hline Thursday & $\bigcirc$ & $\bigcirc$ & $\bigcirc$ & $\bigcirc$ \\
\hline Friday & $\bigcirc$ & $\bigcirc$ & $\bigcirc$ & 0 \\
\hline Saturday & $\bigcirc$ & $\bigcirc$ & $\bigcirc$ & $\bigcirc$ \\
\hline Sunday & $\bigcirc$ & $\bigcirc$ & $\bigcirc$ & $\bigcirc$ \\
\hline
\end{tabular}

Sinus Pain/Congestion

\begin{tabular}{lcccc}
\hline & None & Mild & Moderate & Severe \\
Monday & $\bigcirc$ & $\bigcirc$ & $\bigcirc$ & $\bigcirc$
\end{tabular}




\section{Confidential}

\begin{tabular}{|c|c|c|c|c|}
\hline Tuesday & $\bigcirc$ & $\bigcirc$ & 0 & 0 \\
\hline Wednesday & 0 & $\bigcirc$ & 0 & 0 \\
\hline Thursday & 0 & 0 & 0 & 0 \\
\hline Friday & $\bigcirc$ & $\bigcirc$ & $\bigcirc$ & 0 \\
\hline Saturday & $\bigcirc$ & $\bigcirc$ & $\bigcirc$ & $\bigcirc$ \\
\hline Sunday & $\bigcirc$ & $\bigcirc$ & $\bigcirc$ & 0 \\
\hline \multicolumn{5}{|l|}{ Dry cough } \\
\hline & None & Mild & Moderate & Severe \\
\hline Monday & $\bigcirc$ & $\bigcirc$ & O & $\bigcirc$ \\
\hline Tuesday & $\bigcirc$ & $\bigcirc$ & $\bigcirc$ & $\bigcirc$ \\
\hline Wednesday & $\bigcirc$ & $\bigcirc$ & $\bigcirc$ & $\bigcirc$ \\
\hline Thursday & 0 & 0 & 0 & 0 \\
\hline Friday & 0 & 0 & 0 & $\bigcirc$ \\
\hline Saturday & 0 & $\bigcirc$ & $\bigcirc$ & 0 \\
\hline Sunday & 0 & 0 & 0 & 0 \\
\hline
\end{tabular}

\section{Coughing up Green Phlegm}

\begin{tabular}{lcccc}
\hline Monday & None & Mild & Moderate & Severe \\
Tuesday & 0 & 0 & 0 & 0 \\
Wednesday & 0 & 0 & 0 & 0 \\
Thursday & 0 & 0 & 0 & 0 \\
Friday & 0 & 0 & 0 & 0 \\
Saturday & 0 & 0 & 0 & 0 \\
Sunday & 0 & 0 & 0 & 0 \\
& 0 & 0 & 0 &
\end{tabular}

Coughing up White Phlegm

\begin{tabular}{lcccc}
\hline Monday & None & Mild & Moderate & Severe \\
Tuesday & 0 & 0 & 0 & 0 \\
Wednesday & 0 & 0 & 0 & 0 \\
Thursday & 0 & 0 & 0 & 0 \\
Friday & 0 & 0 & 0 & 0 \\
Saturday & 0 & 0 & 0 & 0 \\
Sunday & 0 & 0 & 0 & 0 \\
& 0 & 0 & 0 & \\
\hline
\end{tabular}

Loss or change to sense of smell 


\section{Confidential}

\begin{tabular}{lcccc} 
& & & Page 9 \\
\hline Monday & None & Mild & Moderate & Severe \\
Tuesday & 0 & 0 & 0 & 0 \\
Wednesday & 0 & 0 & 0 & 0 \\
Thursday & 0 & 0 & 0 & 0 \\
Friday & 0 & 0 & 0 & 0 \\
Saturday & 0 & 0 & 0 & $\bigcirc$ \\
Sunday & 0 & 0 & 0 & 0
\end{tabular}

Loss or change to sense of taste

\begin{tabular}{lcccc}
\hline Monday & None & Mild & Moderate & Severe \\
Tuesday & 0 & 0 & 0 & 0 \\
Wednesday & 0 & 0 & 0 & 0 \\
Thursday & 0 & 0 & 0 & 0 \\
Friday & 0 & 0 & 0 & 0 \\
Saturday & 0 & 0 & 0 & 0 \\
Sunday & 0 & 0 & 0 & 0 \\
& 0 & 0 & 0 & 0
\end{tabular}

Sneezing

\begin{tabular}{lcccc}
\hline Monday & None & Mild & Moderate & Severe \\
Tuesday & 0 & 0 & 0 & 0 \\
Wednesday & 0 & 0 & 0 & 0 \\
Thursday & 0 & 0 & 0 & 0 \\
Friday & 0 & 0 & 0 & 0 \\
Saturday & 0 & 0 & 0 & 0 \\
Sunday & 0 & 0 & 0 & 0
\end{tabular}

Sore Throat

\begin{tabular}{lcccc}
\hline Monday & None & Mild & Moderate & Severe \\
Tuesday & 0 & 0 & 0 & 0 \\
Wednesday & 0 & 0 & 0 & 0 \\
Thursday & 0 & 0 & 0 & 0 \\
Friday & 0 & 0 & 0 & 0 \\
Saturday & 0 & 0 & 0 & 0 \\
Sunday & 0 & 0 & 0 & 0 \\
& 0 & 0 & 0 & 0 \\
\hline
\end{tabular}

Swollen Tonsils 


\section{Confidential}

\begin{tabular}{lcccc} 
& & & Page 10 \\
\hline Monday & None & Mild & Moderate & Severe \\
Tuesday & 0 & 0 & 0 & 0 \\
Wednesday & 0 & 0 & 0 & 0 \\
Thursday & 0 & 0 & 0 & 0 \\
Friday & 0 & 0 & 0 & 0 \\
Saturday & 0 & 0 & 0 & $\bigcirc$ \\
Sunday & 0 & 0 & 0 & 0
\end{tabular}

Swollen Glands (enlarged lymph nodes)

\begin{tabular}{lcccc}
\hline Monday & None & Mild & Moderate & Severe \\
Tuesday & 0 & 0 & 0 & 0 \\
Wednesday & 0 & 0 & 0 & 0 \\
Thursday & 0 & 0 & 0 & 0 \\
Friday & 0 & 0 & 0 & 0 \\
Saturday & 0 & 0 & 0 & $\bigcirc$ \\
Sunday & 0 & 0 & 0 & 0 \\
& 0 & 0 & 0 & \\
\hline
\end{tabular}

Ear pain or change in hearing

\begin{tabular}{lcccc}
\hline Monday & None & Mild & Moderate & Severe \\
Tuesday & 0 & 0 & 0 & 0 \\
Wednesday & 0 & 0 & 0 & 0 \\
Thursday & 0 & 0 & 0 & 0 \\
Friday & 0 & 0 & 0 & 0 \\
Saturday & 0 & 0 & 0 & 0 \\
Sunday & 0 & 0 & 0 & 0
\end{tabular}

\section{Fluid leaking from ear}

\begin{tabular}{lcccc}
\hline Monday & None & Mild & Moderate & Severe \\
Tuesday & 0 & 0 & 0 & 0 \\
Wednesday & 0 & 0 & 0 & 0 \\
Thursday & 0 & 0 & 0 & 0 \\
Friday & 0 & 0 & 0 & 0 \\
Saturday & 0 & 0 & 0 & 0 \\
Sunday & 0 & 0 & 0 & 0 \\
& 0 & 0 & 0 & 0 \\
\hline
\end{tabular}

Shortness of Breath/difficulty breathing 


\section{Confidential}

\begin{tabular}{lcccc}
\hline Monday & None & Mild & Moderate & Severe \\
Tuesday & 0 & 0 & 0 & 0 \\
Wednesday & 0 & 0 & 0 & 0 \\
Thursday & 0 & 0 & 0 & 0 \\
Friday & 0 & 0 & 0 & 0 \\
Saturday & 0 & 0 & 0 & 0 \\
Sunday & 0 & 0 & 0 & 0 \\
& 0 & 0 & 0 & 0 \\
\hline
\end{tabular}

\section{Wheezing}

\begin{tabular}{|c|c|c|c|c|}
\hline & None & Mild & Moderate & Severe \\
\hline Monday & 0 & 0 & $\bigcirc$ & 0 \\
\hline Tuesday & 0 & 0 & 0 & 0 \\
\hline Wednesday & 0 & $\bigcirc$ & 0 & 0 \\
\hline Thursday & 0 & 0 & 0 & 0 \\
\hline Friday & 0 & 0 & 0 & 0 \\
\hline Saturday & 0 & 0 & 0 & 0 \\
\hline Sunday & 0 & 0 & 0 & 0 \\
\hline
\end{tabular}

Chest pain (not changed by breathing or moving)

\begin{tabular}{lcccc}
\hline Monday & None & Mild & Moderate & Severe \\
Tuesday & $\bigcirc$ & 0 & 0 & $\bigcirc$ \\
Wednesday & 0 & 0 & 0 & 0 \\
Thursday & $\bigcirc$ & 0 & 0 & $\bigcirc$ \\
Friday & 0 & 0 & 0 & 0 \\
Saturday & 0 & 0 & 0 & 0 \\
Sunday & 0 & 0 & 0 & 0 \\
& 0 & 0 & 0 & 0
\end{tabular}

Chest pain when breathing in

\begin{tabular}{lcccc}
\hline Monday & None & Mild & Moderate & Severe \\
Tuesday & 0 & 0 & 0 & $\bigcirc$ \\
Wednesday & 0 & 0 & 0 & 0 \\
Thursday & 0 & 0 & 0 & 0 \\
Friday & 0 & 0 & 0 & 0 \\
Saturday & 0 & 0 & 0 & 0 \\
Sunday & 0 & 0 & 0 & 0
\end{tabular}




\section{Confidential}

\section{Other Symptoms - [go_arm_1][hh1_fname] [go_arm_1][hh1_sname]}

[go_arm_1][hh1_fname] [go_arm_1][hh1_sname]: Which eye reläted symptoms did you häve this weeḱ?

Select all that apply

Eye redness

Sticky Eye

Eye pain

Deterioration of eyesight

None of these symptoms

[go_arm_1][hh1_fname] [go_arm_1][hh1_sname]: Where did the rash affect you?

Rash (all over)

Rash (local)

$\square$ None of these symptoms

Where on the body did the rash affect you?

[go_arm_1][hh1_fname] [go_arm_1][hh1_sname]: Which gastrointestinal symptoms did you have?

Select all that apply

$\square$ Diarrhoea (even mild)

$\square$ Vomiting (being sick)

$\square$ Nausea (feeling sick)

$\square$ Abdominal pain (not including menstrual pain)

None of these symptoms

Please identify how severe your symptoms were

Red Eye(s)

\begin{tabular}{|c|c|c|c|c|}
\hline & None & Mild & Moderate & Severe \\
\hline Monday & 0 & 0 & 0 & $\bigcirc$ \\
\hline Tuesday & $\bigcirc$ & 0 & $\bigcirc$ & 0 \\
\hline Wednesday & 0 & 0 & $\bigcirc$ & 0 \\
\hline Thursday & 0 & 0 & 0 & 0 \\
\hline Friday & 0 & 0 & 0 & 0 \\
\hline Saturday & 0 & 0 & 0 & 0 \\
\hline Sunday & 0 & 0 & 0 & 0 \\
\hline
\end{tabular}

Sticky Eye(s)

\begin{tabular}{|c|c|c|c|c|}
\hline & None & Mild & Moderate & Severe \\
\hline Monday & 0 & 0 & 0 & 0 \\
\hline Tuesday & 0 & $\bigcirc$ & 0 & 0 \\
\hline Wednesday & $\bigcirc$ & $\bigcirc$ & $\bigcirc$ & 0 \\
\hline Thursday & 0 & 0 & $\bigcirc$ & 0 \\
\hline Friday & 0 & $\bigcirc$ & $\bigcirc$ & 0 \\
\hline Saturday & $\bigcirc$ & $\bigcirc$ & $\bigcirc$ & $\bigcirc$ \\
\hline Sunday & 0 & 0 & 0 & 0 \\
\hline
\end{tabular}

Eye Pain 


\section{Confidential}

\begin{tabular}{lcccc}
\hline Monday & None & Mild & Moderate & Severe 13 \\
Tuesday & 0 & 0 & 0 & 0 \\
Wednesday & 0 & 0 & 0 & 0 \\
Thursday & 0 & 0 & 0 & 0 \\
Friday & 0 & 0 & 0 & 0 \\
Saturday & 0 & 0 & 0 & 0 \\
Sunday & 0 & 0 & 0 & 0 \\
& 0 & 0 & 0 & 0 \\
\hline
\end{tabular}

\section{Deterioration of eyesight}

\begin{tabular}{|c|c|c|c|c|}
\hline & None & Mild & Moderate & Severe \\
\hline Monday & 0 & 0 & 0 & 0 \\
\hline Tuesday & $\bigcirc$ & $\bigcirc$ & $\bigcirc$ & $\bigcirc$ \\
\hline Wednesday & $\bigcirc$ & $\bigcirc$ & 0 & $\bigcirc$ \\
\hline Thursday & $\bigcirc$ & 0 & 0 & 0 \\
\hline Friday & 0 & 0 & 0 & 0 \\
\hline Saturday & $\bigcirc$ & 0 & $\bigcirc$ & 0 \\
\hline Sunday & 0 & 0 & 0 & 0 \\
\hline
\end{tabular}

Rash - All Over

\begin{tabular}{lcccc}
\hline Monday & None & Mild & Moderate & Severe \\
Tuesday & 0 & 0 & 0 & 0 \\
Wednesday & 0 & 0 & 0 & 0 \\
Thursday & 0 & 0 & 0 & 0 \\
Friday & 0 & 0 & 0 & 0 \\
Saturday & 0 & 0 & 0 & 0 \\
Sunday & 0 & 0 & 0 & 0 \\
& 0 & 0 & 0 &
\end{tabular}

Rash - Local

\begin{tabular}{lcccc}
\hline Monday & None & Mild & Moderate & Severe \\
Tuesday & 0 & 0 & 0 & 0 \\
Wednesday & 0 & 0 & 0 & 0 \\
Thursday & 0 & 0 & 0 & 0 \\
Friday & 0 & 0 & 0 & 0 \\
Saturday & 0 & 0 & 0 & 0 \\
Sunday & 0 & 0 & 0 & 0 \\
& 0 & 0 & 0 & 0 \\
\hline
\end{tabular}

Diarrhoea (even mild) 


\section{Confidential}

\begin{tabular}{|c|c|c|c|c|}
\hline & None & Mild & Moderate & Severe \\
\hline Monday & 0 & $\bigcirc$ & 0 & $\bigcirc$ \\
\hline Tuesday & 0 & 0 & 0 & 0 \\
\hline Wednesday & $\bigcirc$ & 0 & $\bigcirc$ & $\bigcirc$ \\
\hline Thursday & $\bigcirc$ & 0 & $\bigcirc$ & 0 \\
\hline Friday & 0 & 0 & 0 & 0 \\
\hline Saturday & $\bigcirc$ & $\bigcirc$ & $\bigcirc$ & $\bigcirc$ \\
\hline Sunday & 0 & 0 & 0 & 0 \\
\hline
\end{tabular}

Vomiting (being sick)

\begin{tabular}{|c|c|c|c|c|}
\hline & None & Mild & Moderate & Severe \\
\hline Monday & 0 & 0 & $\bigcirc$ & 0 \\
\hline Tuesday & 0 & 0 & 0 & 0 \\
\hline Wednesday & 0 & $\bigcirc$ & 0 & 0 \\
\hline Thursday & 0 & 0 & 0 & 0 \\
\hline Friday & 0 & 0 & 0 & 0 \\
\hline Saturday & 0 & 0 & 0 & 0 \\
\hline Sunday & 0 & 0 & 0 & 0 \\
\hline
\end{tabular}

Nausea (feeling sick)

\begin{tabular}{|c|c|c|c|c|}
\hline & None & $\begin{array}{l}\text { Mild } \\
\end{array}$ & Moderate & Severe \\
\hline Monday & $\bigcirc$ & $\bigcirc$ & 0 & 0 \\
\hline Tuesday & $\bigcirc$ & 0 & 0 & $\bigcirc$ \\
\hline Wednesday & $\bigcirc$ & $\bigcirc$ & $\bigcirc$ & $\bigcirc$ \\
\hline Thursday & $\bigcirc$ & $\bigcirc$ & $\bigcirc$ & $\bigcirc$ \\
\hline Friday & $\bigcirc$ & 0 & 0 & $\bigcirc$ \\
\hline Saturday & $\bigcirc$ & 0 & 0 & $\bigcirc$ \\
\hline Sunday & 0 & 0 & 0 & 0 \\
\hline
\end{tabular}

Abdominal pain (not including menstrual pain)

\begin{tabular}{lcccc}
\hline Monday & None & Mild & Moderate & Severe \\
Tuesday & 0 & 0 & 0 & 0 \\
Wednesday & 0 & 0 & 0 & 0 \\
Thursday & 0 & 0 & 0 & 0 \\
Friday & 0 & 0 & 0 & 0 \\
Saturday & 0 & 0 & 0 & 0 \\
Sunday & 0 & 0 & 0 & 0
\end{tabular}




\section{Confidential}

\section{COVID-19 testing for [go_arm_1][hh1_fname] [go_arm_1][hh1_sname]}

[go_arm_1][hh1_fname] [go_arm_1][hh1_sname]: Have you sought $\bar{C}$ OVID- $\overline{1} \bar{y}$ testing for this îllness via the NHS,

OYes government or your employer?

No

[go_arm_1][hh1_fname] [go_arm_1][hh1_sname]: You said

OYes you tried to get tested for COVID-19 via the NHS,

No government or your employer for this illness. Did you get tested?

[go_arm_1][hh1_fname] [go_arm_1][hh1_sname]: Why did you not get an N $\mathrm{N}$ S test for $\bar{C}$ OVID-19?
I was advised the test was not needed

I am waiting for an NHS test kit to arrive by post

I could not get to a testing centre

I felt better so decided not to get tested

Other - please specify

Other (please specify):

[go_arm_1][hh1_fname] [go_arm_1][hh1_sname]: How long was the gap between your symptoms starting and you having an NHS COVID-19 test?
Same day

Next day

2 days later

3 to 4 days later

5 to 7 days later

More than a week

What was the result of that test?

Positive for COVID-19

Negative for COVID-19

The result was unclear

Still awaiting result

[go_arm_1][hh1_fname] [go_arm_1][hh1_sname]: How long was the gap between your symptoms stärting and you

Same day getting the NHS test results?

$\bigcirc$ Next day

2 days later

3 to 4 days later

5 to 7 days later

$\bigcirc$ More than a week 


\section{Confidential}

\section{Tracing - [go_arm_1][hh1_fname] [go_arm_1][hh1_sname]}

If you had an illness that may be COVID-19 you should follow national advice on reporting this illness and seeking care. We will always have a link to the latest advice on the Virus Watch website.

[go_arm_1][hh1_fname] [go_arm_1][hh1_sname]: Have you reported your illness to any orgañisation other than Virus Watch?

Select all that apply
I did not report my illness to any other organisation

$\square$ Yes to the NHS Contact tracing app

$\square$ Yes to Google/Android contact tracing app

$\square$ Yes to the NHS Test and Trace service

$\square$ Yes to my employer

$\square$ Yes to my GP

$\square$ Yes to the NHS 111 online coronavirus service

$\square$ Yes to the general NHS 111 service

Other (please specify)

Other (please specify)

[go_arm_1][hh1_fname] [go_arm_1][hh1_sname]: Why did you not report your illness?

I did not think it was COVID-19

I didn't feel ill enough to need help

$\square$ I didn't know where to report it

$\square$ I didn't know I was supposed to report it

$\square$ I didn't want to self isolate

$\square$ I didn't want my contacts to have to self isolate

$\square$ I didn't want others to know that I might have COVID-19

[go arm 1][hh1 fname] [go arm 1][hh1 sname]: From the time two days béfore your illness started to now, have you had direct or close contact with anyone OTHER THAN HOUSEHOLD MEMBERS? $\square$ Non-Household Direct Contacts - these are people you had direct physical contact with or with whom you exchanged at least a few words within a 2 metre distance (e.g. a handshake, embracing, kissing, contact sports).

$\square$ Non-Household Close contacts - these are people who were within 2 metres of you for 15 minutes or more but who you did not speak to or touch.

$\square$ I did not have direct or close contact with anybody other than household members

How many people did you have DIRECT CONTACT with other

than household members?

Please enter digits only, e.g. '5' and not 'five'

How many people did you have CLOSE CONTACT with

other than household members?

Please enter digits only, e.g. '5' and not 'five'

[go_arm_1][hh1 fname] [go arm 1][hh1_sname]: Has anyone contactéd you to as $\bar{k}$ about who you have been in contact with prior to or during your illness(contact

tracing)?

Please select all that apply $\square$ No

$\square$ Yes - my GP

Yes - the NHS Test and Trace System

$\square$ Yes - telephone advisory service

$\square$ Yes - through an online form

$\square$ Yes - through an app

$\square$ Yes - through my employer

$\square$ Yes - through my place of education

$\square$ Yes - the local public team

Other - please specify

Other please specify: 


\section{Confidential}

[go_arm_1][hh1_fname] [go_arm_1][hh1_sname]: Was anyone other than a household member asked to self-isolate because of contact with you?
Yes - and I know how many were asked to self isolate

$\bigcirc$ Yes - but I don't know how many were asked to self isolate

Nobody was asked to self isolate

I don't know if anyone was asked to self isolate

How many people other than household contacts were

asked to self-isolate because of contact with you?

Please enter digits only, e.g. '5' and not 'five' 


\section{Confidential}

\section{Health Advice / Consultation - [go_arm_1][hh1_fname] [go_arm_1][hh1_sname]}

[go_arm_1][hh1_fname] [go_arm_1][hh1_sname]: You should follow official national advice about what to do if you have symptoms of COVID-19. We will always include a link to the latest advice on the Virus Watch website.

\begin{tabular}{ll}
\hline [go_arm_1][hh1_fname] [go_arm_1][hh1_sname]: Did the & O Yes \\
illness lead you (or someone else on your behalf) to & ONo
\end{tabular}

No

seek advice about your symptoms this week?

This includes advice from: NHS 111 PharmaciesNurses or

doctorsThe internetFriends and family

Where was the advice or information sought from?

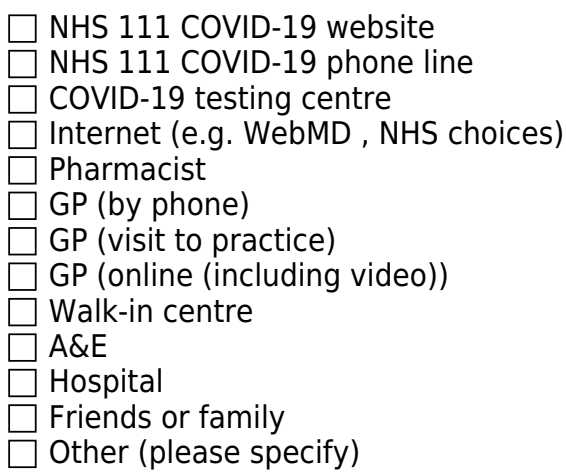

Other (Please Specify):

On which days was the medical advice or information sought from these sources Please check all that that apply on each day

\begin{tabular}{lccccccc}
\hline Internet (e.g. WebMD , NHS & Monday & Tuesday & Wednesday & Thursday & Friday & Saturday & Sunday \\
choices) & $\square$ & $\square$ & $\square$ & $\square$ & $\square$ & $\square$ & $\square$ \\
NHS 111 COVID-19 phone line & $\square$ & $\square$ & $\square$ & $\square$ & $\square$ & $\square$ & $\square$ \\
NHS 111 CoVID-19 website & $\square$ & $\square$ & $\square$ & $\square$ & $\square$ & $\square$ & $\square$ \\
Pharmacist & $\square$ & $\square$ & $\square$ & $\square$ & $\square$ & $\square$ & $\square$ \\
GP (by phone) & $\square$ & $\square$ & $\square$ & $\square$ & $\square$ & $\square$ & $\square$ \\
GP (visit to practice) & $\square$ & $\square$ & $\square$ & $\square$ & $\square$ & $\square$ & $\square$ \\
GP (online (including video)) & $\square$ & $\square$ & $\square$ & $\square$ & $\square$ & $\square$ & $\square$ \\
Walk-in centre & $\square$ & $\square$ & $\square$ & $\square$ & $\square$ & $\square$ & $\square$ \\
COVID-19 testing centre & $\square$ & $\square$ & $\square$ & $\square$ & $\square$ & $\square$ & $\square$ \\
A\&E & $\square$ & $\square$ & $\square$ & $\square$ & $\square$ & $\square$ & $\square$ \\
Hospital & $\square$ & $\square$ & $\square$ & $\square$ & $\square$ & $\square$ & $\square$
\end{tabular}




\section{Confidential}

\section{Medication - [go_arm_1][hh1_fname] [go_arm_1][hh1_sname]}

[go_arm_1][hh1_fname] [go_arm_1][hh1_sname]: Did your symptoms lead to taking any medicines during the

\section{OYes}

survey week?

No

This includes prescribed medicines, medicine brought

at the chemist or shops, or vitamin supplements

On which days did you take the medicines:

Please select all that apply

\begin{tabular}{|c|c|c|c|c|c|c|c|}
\hline & Mon & Tues & Wed & Thurs & Fri & Sat & Sun \\
\hline Paracetamol & $\square$ & $\square$ & $\square$ & $\square$ & $\square$ & $\square$ & $\square$ \\
\hline $\begin{array}{l}\text { Ibuprofen, nurofen, diclofenac, } \\
\text { naproxen or other NSAID }\end{array}$ & $\square$ & $\square$ & $\square$ & $\square$ & $\square$ & $\square$ & $\square$ \\
\hline Aspirin & $\square$ & $\square$ & $\square$ & $\square$ & $\square$ & $\square$ & $\square$ \\
\hline Antibiotics & $\square$ & $\square$ & $\square$ & $\square$ & $\square$ & $\square$ & $\square$ \\
\hline $\begin{array}{l}\text { Cold or flu remedies - over the } \\
\text { counter }\end{array}$ & $\square$ & $\square$ & $\square$ & $\square$ & $\square$ & $\square$ & $\square$ \\
\hline Vitamin Supplements & $\square$ & $\square$ & $\square$ & $\square$ & $\square$ & $\square$ & $\square$ \\
\hline Other (please describe) & $\square$ & $\square$ & $\square$ & $\square$ & $\square$ & $\square$ & $\square$ \\
\hline
\end{tabular}

Please specify: 


\section{Confidential}

\section{Isolation and Infection Control - [go_arm_1][hh1_fname]}

[go_arm_1][hh1_fname] [go_arm_1][hh1_sname]: During the past week, on which days did you:

\begin{tabular}{|c|c|c|c|c|c|c|c|c|}
\hline & Mon & Tues & Wed & Thurs & Fri & Sat & Sun & None \\
\hline Leave the house/flat or garden & $\square$ & $\square$ & $\square$ & $\square$ & $\square$ & $\square$ & $\square$ & $\square$ \\
\hline $\begin{array}{l}\text { Wear a facemask or face } \\
\text { covering outside the home }\end{array}$ & $\square$ & $\square$ & $\square$ & $\square$ & $\square$ & $\square$ & $\square$ & $\square$ \\
\hline $\begin{array}{l}\text { Sleep in a room with no one else } \\
\text { in it? }\end{array}$ & $\square$ & $\square$ & $\square$ & $\square$ & $\square$ & $\square$ & $\square$ & $\square$ \\
\hline $\begin{array}{l}\text { Wear a face mask or face } \\
\text { covering at home? }\end{array}$ & $\square$ & $\square$ & $\square$ & $\square$ & $\square$ & $\square$ & $\square$ & $\square$ \\
\hline $\begin{array}{l}\text { Have a meal with other } \\
\text { members of your household? }\end{array}$ & $\square$ & $\square$ & $\square$ & $\square$ & $\square$ & $\square$ & $\square$ & $\square$ \\
\hline $\begin{array}{l}\text { Watch television with other } \\
\text { members of your household? }\end{array}$ & $\square$ & $\square$ & $\square$ & $\square$ & $\square$ & $\square$ & $\square$ & $\square$ \\
\hline
\end{tabular}

[go arm 1][hh1 fname] [go arm 1][hh1 sname]: During your illnēss, whät best describes your approach to preventing spread of infection in the household?
I did not think my illness was COVID-19, so I did not take any special precautions

I thought it would spread whatever I did so did not take special precautions

I was worried about the illness spreading to others in the household so did what I could to stop this

None of the above

[go_arm_1][hh1_fname] [go_arm_1][hh1_sname]: Since your illness started, on which days did OTHER MEMBERS OF YOŪR HŌUSEHOL̄D:

\begin{tabular}{lcccccccc}
\hline Wear a face mask or face & Mon & Tues & Wed & Thur & Fri & Sat & Sun & None \\
covering when in the same room & $\square$ & $\square$ & $\square$ & $\square$ & $\square$ & $\square$ & $\square \quad$ \\
as you & & & & & & & \\
Leave the house/flat or garden & $\square$ & $\square$ & $\square$ & $\square$ & $\square$ & $\square$ & $\square$ \\
at all & $\square$ & $\square$ & $\square$ & $\square$ & $\square$ & $\square$ & $\square$ & $\square$ \\
$\begin{array}{l}\text { Go to work } \\
\text { Wear a facemask or face } \\
\text { covering outside the home }\end{array}$ & $\square$ & $\square$ & $\square$ & $\square$ & $\square$ & $\square$ & $\square$ & $\square$
\end{tabular}

[go arm_1][hh1 fname] [go arm 1][hh1_sname]: Why did other household members leave the house?

Please select all that apply
They did not think my symptoms were related to COVID-19

$\square$ They did not think there was any need for people without symptoms to stay at home

$\square$ They needed to go out to earn money

$\square$ They needed to go out to an important meeting

$\square$ Other reason 


\section{Confidential}

During your illness, how frequently have you washed your hands thoroughly and regularly with soap and water
Not at all

1 or 2 times a day

3 or 4 times a day

5 or 6 times a day

7 or 8 times a day

9 or 10 times a day

More than 10 times a day

During your illness, on average, how frequently have you (or someone else) disinfected surfaces you might touch?

Such as door knobs or hard surfaces

Several times a day

Daily

Less than daily

Never

[go_arm_1][hh1_fname] [go_arm_1][hh1_sname]: During your illness, how frequently have you:

$\begin{aligned} & \text { Washed your hands after } \\ & \text { blowing your nose, sneezing or } \\ & \text { coughing }\end{aligned}$
$\begin{aligned} & \text { Used tissues when sneezing or } \\ & \text { coughing }\end{aligned}$




\section{Confidential}

\section{Thank You}

Please click submit to continue 